Old Dominion University

ODU Digital Commons

\title{
Attainment of Rigorous Thermodynamic Consistency and Surface Tension in Single-Component Pseudopotential Lattice Boltzmann Models via a Customized Equation of State
}

\author{
Cheng Peng \\ Luis F. Ayala \\ Zhicheng Wang \\ Orlando M. Ayala \\ Old Dominion University, oayala@odu.edu
}

Follow this and additional works at: https://digitalcommons.odu.edu/engtech_fac_pubs

Part of the Engineering Science and Materials Commons, and the Fluid Dynamics Commons

\section{Original Publication Citation}

Peng, C., Ayala, L. F., Wang, Z., \& Ayala, O. M. (2020). Attainment of rigorous thermodynamic consistency and surface tension in single-component pseudopotential lattice Boltzmann models via a customized equation of state. Physical Review E, 101, 17 pp., Article 063309. https://doi.org/10.1103/ PhysRevE.101.063309

This Article is brought to you for free and open access by the Engineering Technology at ODU Digital Commons. It has been accepted for inclusion in Engineering Technology Faculty Publications by an authorized administrator of ODU Digital Commons. For more information, please contact digitalcommons@odu.edu. 


\title{
Attainment of rigorous thermodynamic consistency and surface tension in single-component pseudopotential lattice Boltzmann models via a customized equation of state
}

\author{
Cheng Peng $\odot,{ }^{*}$ Luis F. Ayala $\odot$, and Zhicheng Wang $\odot$ \\ Department of Energy and Mineral Engineering and EMS Energy Institute, The Pennsylvania State University, \\ University Park, Pennsylvania 16802, USA \\ Orlando M. Ayala ${ }^{\circ}$ \\ Department of Engineering Technology, Old Dominion University, 111A Kaufman Hall, Norfolk, Virginia 23529, USA
}

(Received 17 March 2020; accepted 28 May 2020; published 24 June 2020)

\begin{abstract}
The lack of thermodynamic consistency is a well-recognized problem in the single-component pseudopotential lattice Boltzmann models which prevents them from replicating accurate liquid and vapor phase densities; i.e., current models remain unable to exactly match coexisting density values predicted by the associated thermodynamic model. Most of the previous efforts had attempted to solve this problem by introducing tuning parameters, whose determination required empirical trial and error until acceptable thermodynamic consistency was achieved. In this study, we show that the problem can be alternatively solved by properly designing customized equations of state (EOSs) that replace any cubic EOS of choice during the computation of effective mass used in Shan-Chen forces. A two-parameter cubic-shaped customized EOS is introduced. Contrary to previous efforts, customization parameters in the new EOS are nonempirical and are rather derived from solving the integral mechanical stability equation, which neglects the need for any type of tuning for the attainment of rigorous thermodynamic consistency. The proposed approach reduces the errors of the coexisting densities and saturated pressure in the simulation to a maximum of $0.01 \%$ within the liquid-vapor density ratio range from $O(1)$ to $O\left(10^{4}\right)$, which had not been achieved in any of the previous tuning-based efforts. A straightforward way for achieving the desired surface tension via the customized EOS is also provided.
\end{abstract}

DOI: 10.1103/PhysRevE.101.063309

\section{INTRODUCTION}

In the past two decades, pseudopotential (PP) lattice Boltzmann (LB) models [1,2] have been widely used to simulate a variety of multiphase problems involving both pure and multicomponent immiscible fluids [3-5]. The major advantage of PP LB models, which explains their frequent deployments in multiphase applications, is their conceptual simplicity and computational efficiency [4]. In these PP LB models, phase separation and surface tension forces that give rise to capillary pressure are realized by defining a single fully discretized body force, known as the Shan-Chen force [1,2]. For LB simulations to reproduce realistic (nonideal gas) fluid properties, thermodynamically meaningful cubic equations of state (EOSs) are incorporated into the definition of Shan-Chen forces [6]. This is realized through coupling thermodynamic pressure values generated by a cubic EOS with the modified hydrodynamic pressure definition derived after the addition of Shan-Chen forces into the Navier-Stokes equations (NSEs) [6]. However, this treatment is known to typically lead to thermodynamic inconsistencies; i.e., LB simulation results for coexisting liquid and vapor density values deviate from the actual values predicted by the thermodynamic model-with the largest deviations occurring in vapor phase densities [7,8]. The lack of thermodynamic consistency in PP LB models is

*czp341@psu.edu one of the major deficiencies of the PP LB models for pure substances applications [3,5]. It is also recognized as a main cause for the numerical instability when PP LB models are deployed in applications involving large liquid-vapor density ratios [8].

In the past, a number of efforts were devoted to better understand and try to alleviate the thermodynamic inconsistency problem of PP LB models. He and Doolen compared the mechanical stability condition associated to PP LB models against the requirements of Maxwell's equal area rule and concluded that thermodynamic consistency cannot be satisfied by PP LB models [9]. Later, Shan [10] pointed out the failure of accounting for the discrete nature of LB simulations in $\mathrm{He}$ and Doolen's derivation of mechanical stability conditions. The corrected mechanical stability condition derived by Shan indicated that thermodynamic consistency in PP LB models could still be achieved if the effective mass $\psi$ used to define the Shan-Chen force was made proportional to $\exp (-1 / \rho)$, where $\rho$ is the local fluid density [10]. As discussed later in Sec. II, it should be noted that such a requirement for thermodynamic consistency would always be violated if a cubic EOS is incorporated into PP LB models to define the effective mass $\psi$, given that the effective mass would not be related to density through an exponential relationship.

So far, multiple efforts have been devoted to address the problem of thermodynamic inconsistency in PP LB models [7,8,11-14]. These efforts are reviewed in detail in Sec. III. While these previous efforts take different approaches towards 
achieving the same ultimate goal, a common feature is the introduction of tuning parameters, whose proper values are empirically determined until thermodynamic consistency is restored in PP LB models. Unfortunately, the reported values of tuned parameters cannot be generalized, since they are invariably case specific and only available for the specific choices of EOS used in those studies $[8,14,15]$. From the users' point of view, when a different cubic EOS and different conditions are adopted, a time-consuming trial-and-error process remains unavoidable in order to quantify the proper value of tuning parameters. And more problematically, even if the values of tuning parameters provided in those studies are used for the same conditions and same EOS, certain amount of deviations still result in the coexisting liquid-vapor densities, especially when liquid-vapor density ratios are large [8,14]. For applications with high accuracy requirements, the available values of tuning parameters cannot be used without further refinement.

The main purpose of the current study is to identify a rigorous approach to restore full thermodynamic consistency in PP LB models, for any arbitrary choices of cubic EOS, and without the necessity of tuning or trial and error. This can be achieved by properly customizing the van der Waals loop of the cubic EOS within equilibrium bubble point and dew point conditions during a liquid-vapor transition. The customized EOS contains two calibration parameters; both can be analytically obtained without any need for tuning. With the current approach, precise thermodynamic consistency, i.e., with less than $0.01 \%$ deviation of the coexisting densities and saturated pressure for the liquid-vapor density ratio ranging from $O(1)$ to $O\left(10^{4}\right)$, can be achieved with reasonable interface thicknesses. Moreover, the current approach of customizing EOS also allows the resulting surface tension from PP LB models to remain adjustable within given coexisting densities and saturated pressure.

The rest of this document is arranged as follows. In Sec. II, we briefly describe PP LB models and their associated problems with thermodynamic consistency. Previous efforts to improve the thermodynamic consistency are also reviewed in Sec. III. Then in Sec. IV, we propose and derive the proper customization of the cubic EOS within the liquid-vapor transition (van der Waals loop) so that not only a proper pressuredensity relationship is maintained in bulk phase regions, but also precise thermodynamic consistency is achieved across phases. Discussions and comparisons between the proposed approach and previous efforts are presented in Sec. V. In Sec. VI, we discuss the feature of adjusting the surface tension to desired values using the customized EOS. Finally, main conclusions of this study are summarized in Sec. VII.

\section{THERMODYNAMIC INCONSISTENCY IN PSEUDOPOTENTIAL LB MODELS}

The governing equation of the lattice Boltzmann method (LBM), i.e., the lattice Boltzmann equation (LBE) with a single-relaxation time, reads

$$
\begin{aligned}
f_{\alpha}(\mathbf{x} & \left.+\mathbf{e}_{\alpha} \delta t, t+\delta t\right)-f_{\alpha}(\mathbf{x}, t) \\
& =-\frac{1}{\tau}\left[f_{\alpha}(\mathbf{x}, t)-f_{\alpha}^{(e q)}(\mathbf{x}, t)\right]+F_{\alpha}(\mathbf{x}, t),
\end{aligned}
$$

where $f_{\alpha}$ is the distribution function associated with the lattice velocity $\mathbf{e}_{\alpha}, f_{\alpha}^{(e q)}$ is the corresponding equilibrium distribution function, $\mathbf{x}$ is the spatial coordinate, $t$ is the current time, and $\delta t$ is the time interval between two consecutive time steps. $\tau$ is the unique nondimensional relaxation time of the distribution functions, which is related to the kinematic viscosity $v$ of the fluid as

$$
v=\left(\tau-\frac{1}{2}\right) c_{s}^{2} \delta t
$$

where $c_{s}$ is the speed of sound in the LBM that is associated with the lattice type. $F_{\alpha}$ is the forcing term representing the effect of the external body force on $f_{\alpha}$. In a standard LB model, the equilibrium distribution function $f_{\alpha}^{(e q)}$ and the forcing term $F_{\alpha}$ are expressed in terms of the macroscopic quantities as [16]

$$
\begin{aligned}
f_{\alpha}^{(e q)} & =\rho w_{\alpha}\left[1+\frac{e_{\alpha i} u_{i}}{c_{s}^{2}}+\frac{\left(e_{\alpha i} e_{\alpha j}-c_{s}^{2} \delta_{i j}\right) u_{i} u_{j}}{2 c_{s}^{4}}\right] \\
F_{\alpha} & =\left(1-\frac{1}{2 \tau}\right) w_{\alpha}\left[\frac{e_{\alpha i} F_{i}}{c_{s}^{2}}+\frac{\left(e_{\alpha i} e_{\alpha j}-c_{s}^{2} \delta_{i j}\right) u_{j} F_{i}}{c_{s}^{4}}\right] \delta t
\end{aligned}
$$

where $\rho, u_{i}$, and $F_{i}$ are the local fluid density, velocity, and external body force, respectively; $w_{\alpha}$ is the weighting factor that depends on the specific lattice chosen in the simulation. Equation (3b) is the well-known Guo et al. forcing scheme [16] that ensures a nonuniform body force field is discretized in the LBE with a second-order accuracy. In the LBM, the hydrodynamic quantities $\rho$ and $u_{i}$ are calculated from the distribution functions as

$$
\rho=\sum_{\alpha} f_{\alpha}, \quad \rho u_{i}=\sum_{\alpha} f_{\alpha} e_{\alpha i}+\frac{1}{2} F_{i} \delta t .
$$

PP LB models facilitate multiphase flow simulations through the introduction of an external body force, known as the Shan-Chen force, which is defined as $[1,2]$

$$
F_{i}(\mathbf{x})=-G \psi(\mathbf{x}) \sum_{\beta} W_{\beta} \psi\left(\mathbf{x}+\mathbf{e}_{\beta} \delta t\right) e_{\beta i},
$$

where $G$ is the forcing intensity, $\psi(\mathbf{x})$ and $\psi\left(\mathbf{x}+\mathbf{e}_{\beta} \delta t\right)$ are the effective mass at the current location and its neighboring locations, respectively, and $W_{\beta}$ is the weighting factor which determines the relative contribution from each neighboring location to the external body $F_{i}$. While not necessary, $\mathbf{e}_{\beta}$ and $W_{\beta}$ can be chosen as the lattice velocities and the lattice weighting, i.e., $\mathbf{e}_{\alpha}$ and $w_{\alpha}$, respectively, out of convenience. This choice also ensures the calculation of the Shan-Chen force and the distribution of this force among distribution functions has the same order of isotropy, which strikes a balance between suppression of spurious currents and computational cost [17]. It is therefore adopted in this study as well.

With the addition of the external body force $F_{i}$ in Eq. (5) into the NSEs, the fluid pressure or hydrodynamic pressure of the vapor and liquid phases, $p_{\mathrm{H}}$, is related to the phase density $\rho$ and the effective mass $\psi$ as [6]

$$
p_{\mathrm{H}}=c_{s}^{2} \rho+\frac{1}{2} G \delta t c_{s}^{2} \psi^{2} .
$$

To ensure that this fluid pressure is consistent with fluid thermodynamics, Yuan and Schaefer [6] incorporated 
thermodynamic pressure from a cubic EOS into the definition of $\psi$, which gives

$$
\psi=\sqrt{\frac{2\left(p_{\mathrm{EOS}}-c_{s}^{2} \rho\right)}{G c_{s}^{2} \delta t}}
$$

where $p_{\mathrm{EOS}}$ is a thermodynamic pressure at a given density that is calculated from a selected cubic EOS. The main benefit of using Eq. (7) to define $\psi$ is that it ensures that fluid pressure converges to the thermodynamic pressure, as prescribed by the cubic EOS, throughout bulk regions where single-phase fluids are found (either as liquid or vapor). This definition of $\psi$ has also been found beneficial for numerical stability compared to its original definitions $[3,6]$. It should also be noted that when $\psi$ is calculated with Eq. (7), the forcing intensity $G$ no longer controls the magnitude of the external force $F_{i}$, but it is still needed to ensure that the entire term under the square root is non-negative.

The problem with Yuan and Schaefer's way of defining $\psi$ is that it has been found to lead to thermodynamic discrepancies between LB results and cubic EOS predictions across phases in coexisting equilibrium $[7,8,15]$. As a result, LB simulation results for coexisting two-phase densities and saturated pressure typically deviate from their true thermodynamic values, as predicted by the Maxwell equal area rule applied to the cubic EOS of choice [18]. The reason behind these thermodynamic discrepancies has been analyzed in a few studies [8-10]. When a PP LB model with nearest-neighbor interactions is applied to a two-phase system separated by a flat interface, it yields a mechanical stability condition (or chemical balance equation as referred to by some authors), which reads [10]

$$
\begin{aligned}
& \int_{\rho_{V}}^{\rho_{L}}\left[c_{s}^{2} \rho_{L}+\frac{1}{2} c_{s}^{2} G \delta t \psi^{2}\left(\rho_{L}\right)-c_{s}^{2} \rho-\frac{1}{2} c_{s}^{2} G \delta t \psi^{2}(\rho)\right] \frac{\psi^{\prime}}{\psi} d \rho \\
& \quad=0
\end{aligned}
$$

where $\psi^{\prime}=d \psi / d \rho$, and $\rho_{L}$ and $\rho_{V}$ are the fluid densities in the liquid and vapor bulk phases predicted by PP LB models.

Comparing the mechanical stability condition in Eq. (8) with the Maxwell equal area rule [18]

$$
\int_{\tilde{\rho}_{V}}^{\tilde{\rho}_{L}}\left[p_{\operatorname{EOS}}\left(\tilde{\rho}_{L}\right)-p_{\operatorname{EOS}}(\rho)\right] \frac{1}{\rho^{2}} d \rho=0,
$$

where $\tilde{\rho}_{L}$ and $\tilde{\rho}_{V}$ are the coexisting liquid and vapor phase densities predicted by the Maxwell equal area rule, it becomes evident that a sufficient but not necessary condition to achieve thermodynamic consistency, i.e., $\rho_{L}=\tilde{\rho}_{L}$ and $\rho_{V}=\tilde{\rho}_{V}$, is

$$
p_{\mathrm{EOS}}(\rho)=c_{s}^{2} \rho+\frac{1}{2} c_{s}^{2} G \delta t \psi^{2}(\rho), \quad \psi \propto \exp \left(-\frac{1}{\rho}\right) .
$$

Note that when $\psi$ is defined through Yuan and Schaefer's approach [6] via a cubic EOS, the first requirement is automatically satisfied, but the second requirement is usually violated. As there is only one unknown $(\psi)$ available to enforce these requirements in a typical PP LB model, these two conditions are unlikely to be satisfied simultaneously.

Historically, Eq. (8) was first derived by Shan [10]. However, Shan was not the first one to recognize there was a problem of thermodynamic inconsistency in PP LB models.
He and Doolen [9] Taylor-expanded the Shan-Chen force in Eq. (5), then absorbed the force into the pressure gradient term in the NSE, and eventually reached a mechanical stability condition that reads

$$
\begin{aligned}
& \int_{\rho_{V}}^{\rho_{L}}\left[c_{s}^{2} \rho_{L}+\frac{1}{2} c_{s}^{2} G \delta t \psi^{2}\left(\rho_{L}\right)-c_{s}^{2} \rho-\frac{1}{2} c_{s}^{2} G \delta t \psi^{2}(\rho)\right] \frac{\psi^{\prime}}{\psi^{2}} d \rho \\
& \quad=0 .
\end{aligned}
$$

By comparing Eq. (11) to Eq. (9), He and Doolen concluded that only when $\psi \propto \rho$ can PP LB models satisfy thermodynamic consistency [9]. Later, Shan pointed out that He and Doolen's derivation did not account for the discrete nature of the LBM and thus it could not reflect the actual situation in the simulations [10]. Equation (8) was then derived to be the correct mechanical stability condition when the discrete nature of the LBM is taken into account.

The cross examination of Eq. (8) and Eq. (9) does provide a clear view that allows one to identify the reason behind thermodynamic inconsistencies in PP LB models. However, since the thickness of the interface is always finite in the actual LB simulations, i.e., the transition of fluid density from $\rho_{V}$ to $\rho_{L}$ always happens across a number of (preferably few) discrete points, the presence of a continuous integral in Eq. (8) may still omit some parts of the "discrete nature" of the LBM. By fully considering the discrete nature of the LBM, we can show that the mechanical stability criteria would read as

$$
\sum_{j} \frac{c_{s}^{2} \rho_{L}+\frac{1}{2} c_{s}^{2} G \delta t \psi^{2}\left(\rho_{L}\right)-c_{s}^{2} \rho(j)-\frac{1}{2} c_{s}^{2} G \delta t \psi^{2}(j)}{\psi(j)}=0,
$$

where the summation is over the discrete grid points ( $j$ being their indices) within the interface, which is rigorously followed when a PP LB simulation in a flat interface case reaches its steady state. In this document, we refer conditions in the form of Eq. (8) as integral mechanical stability conditions, and conditions in the form of Eq. (12) as discrete mechanical stability conditions. As we show later, when the Shan-Chen force is defined with its original definition in Eq. (5) and discretized with Guo et al.'s forcing scheme, the resulting integral mechanical stability condition turns out to be an extremely good approximation of its discrete counterpart. This feature will be taken advantage of by the current study to achieve precise thermodynamic consistency in PP LB models. In Appendix A, we provide a detailed derivation for both the integral and discrete mechanical stability conditions with nearest-neighbor interactions, i.e., Eq. (8) and Eq. (12). The derivation of Eq. (8) is different from Shan's derivation in Ref. [10] but it leads to the same result.

\section{A REVIEW OF PREVIOUS EFFORTS ON IMPROVING THERMODYNAMIC CONSISTENCY OF PSEUDOPOTENTIAL LB MODELS}

Different types of remedies to improve thermodynamic consistency problems of pseudopotential LB models have been proposed in the literature. These remedies can be roughly classified into three categories: (1) modification of the Shan-Chen force $[13,14,19]$, (2) modification of the forcing scheme of the LBE $[8,11,12]$, and (3) modification of the 
EOSs of choice during effective mass computations $\psi$ [7]. Representative works for each category are briefly reviewed here.

Kupershtokh et al. [19] proposed to replace the definition of the Shan-Chen force [Eq. (5)] with

$$
\begin{aligned}
F_{i}= & -\beta G \psi(\mathbf{x}) \sum_{\alpha} w_{\alpha} \psi\left(\mathbf{x}+\mathbf{e}_{\alpha} \delta t\right) e_{\alpha i} \\
& -\frac{1-\beta}{2} G \sum_{\alpha} w_{\alpha} \psi^{2}\left(\mathbf{x}+\mathbf{e}_{\alpha} \delta t\right) e_{\alpha i},
\end{aligned}
$$

where $\beta$ is a tuning parameter introduced to be computed by trial and error until the accuracy of the simulated two-phase densities is improved.

Alternatively, Kharmiani et al. [14] modified the ShanChen force as

$$
F_{i}=-G \psi(\mathbf{x}) \sum_{\alpha} w_{\alpha} \psi\left(\mathbf{x}+\mathbf{e}_{\alpha} \delta t\right) e_{\alpha i}+\lambda \frac{G \delta t c_{s}^{2}}{12} \partial_{i}\left(\left|\partial_{k} \psi\right|^{2}\right) .
$$

The second term in Eq. (14) is a correction term with $\lambda$ being the tuning parameter subject to trial-and-error determination until thermodynamic inconsistency can be reduced significantly.

Khajepor and Chen [13] proposed a multipseudopotential interaction scheme, where the Shan-Chen force was designed as

$$
F_{i}=\sum_{j=1}^{n}-G_{j} \psi_{j}(\mathbf{x}) \sum_{\alpha} w_{\alpha} \psi_{j}\left(\mathbf{x}+\mathbf{e}_{\alpha} \delta t\right) e_{\alpha i},
$$

where the total force consists of $n$ parts, each defined with separated $G_{j}$ and $\psi_{j}$. With this multipseudopotential interaction force, the hydrodynamic pressure in the vapor and liquid phases becomes

$$
p_{H}=c_{s}^{2} \rho+\frac{1}{2} \delta t c_{s}^{2} \sum_{j}^{n} G_{j} \psi_{j}^{2} .
$$

The effective mass $\psi_{j}$ in this scheme is defined as

$$
\psi_{j}=\left(\frac{\rho}{\lambda_{j} \epsilon_{j}+C_{j} \rho}\right)^{1 / \epsilon_{j}},
$$

where $\lambda_{j}, \epsilon_{j}$, and $C_{j}$ are constants that can be directly determined by matching each term in the resulting hydrodynamic pressure in Eq. (17) with a part in the target $p_{\text {EOS. While this }}$ scheme no longer requires tuning for the newly introduced parameters, significant errors of thermodynamic discrepancies are still observed when the liquid-vapor density ratios were large [13]. The above three attempts all belong to the category of attempting to revise the definition of the Shan-Chen force in order to improve the thermodynamic consistency of PP LB models.

Alternatively, Li et al. [8] proposed using a modified forcing scheme in the LBE to restore thermodynamic consistency. With the Shan-Chen force still defined by Eq. (5), Li et al. revised the forcing scheme in the LBE [i.e., Eq. (3b)] as

$$
F_{\alpha}=\left(1-\frac{1}{2 \tau}\right) w_{\alpha}\left[\frac{e_{\alpha i} F_{i}}{c_{s}^{2}}+\frac{\left(e_{\alpha i} e_{\alpha j}-c_{s}^{2} \delta_{i j}\right) u_{j}^{\prime} F_{i}}{c_{s}^{4}}\right],
$$

where $u_{j}^{\prime}=u_{j}+\sigma F_{j} /\left[(\tau-0.5) c_{s}^{2} \psi^{2}\right]$, and $\sigma$ is a tuning parameter that needs to be properly defined for thermodynamic consistency. A moment-based expression of this forcing scheme could be found in Ref. [11] when the multirelaxation-time LBE is adopted. Xu et al. further extended this idea to a D3Q15 lattice model for three-dimensional applications [12].

The above two categories of remedies determine $\psi$ in terms of cubic EOSs via Eq. (7). A third category of remedies seeks to replace cubic EOSs with some modified EOS version that can improve thermodynamic consistency in PP LB models. To the best of our knowledge, Colosqui et al. [7] was the first to deploy this idea by proposing the use of a piecewise linear EOS [7]:

$$
p(\rho)= \begin{cases}\rho \theta_{V} & \text { if } \rho \leqslant \rho_{1} \\ \rho_{1} \theta_{V}+\left(\rho-\rho_{1}\right) \theta_{M} & \text { if } \rho_{1} \leqslant \rho \leqslant \rho_{2} \\ \rho_{1} \theta_{V}+\left(\rho_{2}-\rho_{1}\right) \theta_{M}+\left(\rho-\rho_{2}\right) \theta_{L} & \text { if } \rho>\rho_{2},\end{cases}
$$

where $\theta_{V}=\sqrt{(d p / d \rho)_{V}}, \theta_{L}=\sqrt{(d p / d \rho)_{L}}$ are the speed of sound of the coexisting vapor and liquid phases, respectively, and $\theta_{M}$ is the slope of the unstable branch of the customized EOS, which has a negative value. Parameters $\rho_{1}$ and $\rho_{2}$ are determined via solving the mechanical balance equation

$$
\int_{\tilde{\rho}_{V}}^{\tilde{\rho}_{L}} d p=\left(\rho_{1}-\tilde{\rho}_{V}\right) \theta_{V}+\left(\rho_{2}-\rho_{1}\right) \theta_{M}+\left(\tilde{\rho}_{L}-\rho_{2}\right) \theta_{L}=0,
$$

and the chemical balance equation

$$
\begin{aligned}
\int_{\tilde{\rho}_{V}}^{\tilde{\rho}_{L}} \frac{1}{\rho} d p= & \ln \left(\rho_{1} / \tilde{\rho}_{V}\right) \theta_{V}+\ln \left(\rho_{2} / \rho_{1}\right) \theta_{M} \\
& +\ln \left(\tilde{\rho}_{L} / \rho_{2}\right) \theta_{L}=0 .
\end{aligned}
$$

The modified EOS in Eq. (19) is not able to achieve thermodynamic consistency on its own, as the simulation results for the two-phase densities, $\rho_{V}$ and $\rho_{L}$, still deviated significantly from their targets $\tilde{\rho}_{V}$ and $\tilde{\rho}_{L}$, respectively. In order to restore thermodynamic consistency, a pressure correction $(\delta p)$ needs to be applied to the pressure resulting from Eq. (19) before being used by Eq. (7) to determine $\psi$. This pressure correction $\delta p$ is defined as [7]

$$
\delta p\left(\rho, \alpha_{1}, \alpha_{2}\right)= \begin{cases}0 & \text { if } \rho \leqslant \rho_{1} \\ p_{M} \frac{\rho-\rho_{1}}{\rho_{M}-\rho_{1}} & \text { if } \rho_{1}<\rho \leqslant \rho_{M} \\ p_{M} \frac{\rho-\rho_{2}}{\rho_{M}-\rho_{2}} & \text { if } \rho_{M}<\rho \leqslant \rho_{2} \\ 0 & \text { if } \rho>\rho_{2},\end{cases}
$$

where $p_{M}=\alpha_{1}\left(\rho_{1}-\rho_{2}\right) \theta_{M}$ and $\rho_{M}=\rho_{1}+\alpha_{2}\left(\rho_{2}-\rho_{1}\right)$ are two newly introduced variables that are made functions of two tuning parameters $\alpha_{1}$ and $\alpha_{2}$. Essentially, Colosqui et al.'s approach tunes these two parameters as well as $\theta_{M}$ in Eq. (19) to achieve thermodynamic consistency [7].

The design by Colosqui et al. has a few possible shortcomings. First, for single-phase regions, i.e., when $\rho \leqslant \tilde{\rho}_{V}$ and $\rho \geqslant \tilde{\rho}_{L}$, Colosqui et al.'s customized EOS assumes linear relationships between density and pressure, which does not fully honor the real fluid behavior. One of the main reasons that cubic EOSs are often employed in PP 
LB models is that they describe the nonlinear physical relationship between pressure and fluid density within the single-phase branches, which should be enforced within bulk regions away from interfaces. Second, Colosqui et al.'s EOS modification relies on three tuning parameters: $\theta_{M}$ in Eq. (19), and $\alpha_{1}$ and $\alpha_{2}$ in Eq. (22). While Colosqui et al. did suggest fixing $\theta_{M}$ and $\alpha_{1}$, and automatically tune $\alpha_{2}$ through a computer program, proper guesses of $\theta_{M}$ and $\alpha_{1}$ are still needed and rigorous thermodynamic consistency is yet not fully attained.

To summarize previous efforts of improving thermodynamic consistency in PP LB models, with the exception of the work of Khajepor and Chen [13], all available methods rely on tuning newly introduced parameters in order to attempt thermodynamic consistency. There are two significant and common drawbacks in these tuning-based efforts. First, proper values of the tuning parameters are only available for the specific and narrow conditions chosen by the original authors, e.g., for their specific choice of EOS and for certain temperature range selections $[8,14]$. From the user's point of view, once different conditions are encountered, a time-consuming trial-and-error process is required in order to define all tuning parameters. Second, even when recommended values of the tuning parameters are available, simulation results for the vapor branch density could still contain up to $10 \%$ or higher deviations from their actual values at large density ratios, which may not be acceptable for certain applications. The method of Khajepor and Chen [13], on the other hand, does not require tuning but can still lead to significant errors of thermodynamic inconsistency for applications where liquidvapor density ratios are large. As a result, developing a method that can enforce precise thermodynamic consistency without the necessity for empirical tuning is the major motivation driving the current study.

\section{ACHIEVING RIGOROUS THERMODYNAMIC CONSISTENCY WITH A PROPERLY CUSTOMIZED CUBIC EOS}

A cubic EOS is an equation that describes the thermodynamic state of a substance and prescribes a continuous density(volume)-pressure-temperature relationship for liquid and vapor phases and their phase transitions. As a result, it can be deployed to calculate single-phase vapor and single-phase liquid properties and also to predict locations of the bubble and dew points at which phase transitions happen. While the density(volume)-pressure-temperature relationship prescribed by the cubic EOSs should be fully honored for all bulk phase behavior (e.g., liquid and vapor branches), there remains room for customization of EOS behavior during phase transitions for the purpose of improving the thermodynamic consistency performance of PP LB models. This room for customization stems from two aspects.

First, while a cubic EOS is defined continuously at any given density, its predictions are always physically meaningful when applied to thermodynamically stable phases (either single-phase liquid or single-phase vapor). In Fig. 1, the single-phase branches of a cubic EOS for a pure substance at a given temperature (below critical temperature) are marked by two black solid lines. The section in between the two single-

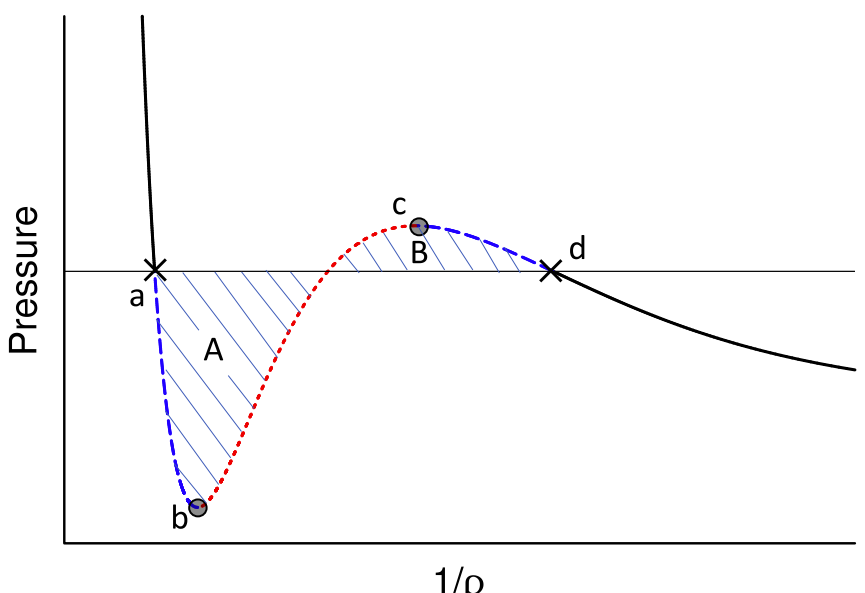

FIG. 1. A cubic equation of state.

phase branches, i.e., the curve "a-b-c-d" in Fig. 1, known as a van der Waals loop, is a well-known artifact of mean-field molecular theories applied to fluids undergoing vapor-liquid transitions (the case of cubic EOSs deployed at subcritical temperatures). For pure substances, true equilibrium phase transition with flat interfaces takes place at constant pressure and temperature, i.e., along the straight line discontinuously connecting points "a" and "d," but cubic EOSs connect these two end points of the liquid-vapor transition continuously with an S-shaped van der Waals loop. Section "b-c" of the van der Waals loop is the most problematic with regards to true fluid behavior, since it leads to negative fluid compressibilities (i.e., fluid expands with increased fluid pressure), which violates the second law of thermodynamics for phase stability. While segment "b-c" of any cubic loop is always deemed unphysical and must be rejected, segments "a-b" and "c-d" may only be realized in real systems when metastable states are present. More generally, the classical thermodynamic theory of equilibrium generally asserts that none of these states within the van der Waals loop is to be observed in equilibrium two-phase systems with flat interfaces. Therefore, for the purpose of representing equilibrium thermodynamics in these two-phase systems with flat interfaces, it is feasible to replace the original "a-b-c-d" loop of any cubic EOS with a customized loop.

Second, fluid densities associated with van der Waals loops only exist in LB simulations at the few points found within a (desirably narrow) discretized flat interface. The ultimate goal of PP LB simulations is to obtain thermodynamic meaningful fluid properties in bulk fluid regions. Enforcing any particular pressure-density relationship at interface nodes is only of concern for surface tension purposes, which is covered in Sec. VI, but it is not for the bulk regions, which represent the large majority of the computational domain. In this work, the use of a customized EOS to replace the original cubic EOSs at interfaces is only needed for the definition of the effective mass $\psi$ and proper calibration of the associated Shan-Chen forces. It should be kept in mind that $\psi$ is not a physical quantity but a numerical design meant to enable multiphase flow simulations and to drive proper fluid separation. Once proper fluid separation has been accomplished with properly calibrated Shan-Chen forces, the original cubic EOS of choice should still be used to resolve the pressure-density 
relationship whenever needed. In other words, the proposed customized EOS loop is only to be used for "under-the-hood" calculations for Shan-Chen forces at interfaces, and it is not meant to be deployed anywhere where actual thermodynamic information is required.

Based on the above considerations, we propose the following customized cubic EOS for PP LBM simulations:

$$
\tilde{p}(\rho)= \begin{cases}p_{\operatorname{EOS}}(\rho) & \text { if } \rho \leqslant \tilde{\rho}_{V} \\ \tilde{p}_{0}+\theta\left(\rho-\tilde{\rho}_{V}\right)\left(\rho-\tilde{\rho}_{L}\right)\left(\rho-\tilde{\rho}_{M}\right) & \text { if } \tilde{\rho}_{V}<\rho<\tilde{\rho}_{L} \\ p_{\operatorname{EOS}}(\rho) & \text { if } \rho \geqslant \tilde{\rho}_{L},\end{cases}
$$

where $\tilde{p}_{0}$ is the saturation pressure corresponding to the prevailing temperature which defines fluid density at the bubble (saturated liquid density) and dew (saturated vapor density) points, i.e., $\tilde{p}_{0}=p_{\mathrm{EOS}}\left(\tilde{\rho}_{L}\right)=p_{\mathrm{EOS}}\left(\tilde{\rho}_{V}\right)$; $\tilde{\rho}_{M}$ and $\theta$ are the middle root and the amplitude of the customized EOS loop, respectively. $\tilde{p}_{0}, \tilde{\rho}_{L}$, and $\tilde{\rho}_{V}$ can be obtained from the Maxwell equal area rule applied to the cubic EOS of choice at the given reduced temperature. Contrary to the design of Colosqui et al. [7], the customization in Eq. (23) retains the cubic character of the EOS and is restricted to the van der Waals loop and thus only to the calculation of $\psi$ at interfaces. Outside the van der Waals loop, $\rho \leqslant \tilde{\rho}_{V}$ and $\rho \geqslant \tilde{\rho}_{L}$, the customized pressure rigorously follows the thermodynamic pressure from the cubic EOS, as thermodynamically required. Inside the van der Waals loop, the customized pressure consists of two parts: a constant saturated pressure $\tilde{p}_{0}$ that ensures the pressure is continuous at the bubble and dew points, and a cubic function that is necessary for phase separation. In contrast with the design of Colosqui et al. [7], the two customization variables in the proposed EOS, $\theta$ and $\rho_{M}$, can be directly defined and constrained. As discussed later in Sec. VI, $\theta$ controls the amplitude of the customized loop and can be constrained by the desired value of surface tension at the interface. When interface tension is not of essential concern, one can define $\theta$ as

$$
\theta=\left.\frac{1}{\left(\tilde{\rho}_{V}-\tilde{\rho}_{M}\right)\left(\tilde{\rho}_{V}-\tilde{\rho}_{L}\right)} \frac{d p_{\mathrm{EOS}}}{d \rho}\right|_{\rho=\tilde{\rho}_{V}},
$$

which results in the compressibility of the customized EOS $d \tilde{p} / d \rho$ being continuous at the dew point, $\rho=\tilde{\rho}_{V}$. With Eq. (24), the continuity of the compressibility at the bubble point $\rho=\tilde{\rho}_{L}$ is not constrained. The middle density root of the customized cubic function $\tilde{\rho}_{M}$ is to be found to ensure that resulting Shan-Chen forces lead to proper fluid separation based on rigorous thermodynamic consistency. As mentioned earlier, the integral mechanical stability condition, Eq. (8), is the equation to be satisfied by the actual two-phase coexisting densities $\rho_{L}$ and $\rho_{V}$ for a PP LB simulation with a flat interface. When the two-phase densities $\rho_{L}$ and $\rho_{V}$ from the simulation are fully consistent with their corresponding values predicted by the Maxwell equal area rule, $\tilde{\rho}_{L}$ and $\tilde{\rho}_{V}$, a fully thermodynamically consistent PP LB model would be achieved. Obviously, the target $\tilde{\rho}_{M}$ becomes the root of the integral equation

$$
\tilde{\rho}_{M}=\operatorname{root}\left[\int_{\tilde{\rho}_{V}}^{\tilde{\rho}_{L}}\left[\tilde{p}_{0}-\tilde{p}(\rho)\right] \frac{\psi^{\prime}}{\psi} d \rho=0\right] .
$$

Recall that $\psi$ and $\psi^{\prime}$ are

$$
\begin{aligned}
\psi & =\sqrt{\frac{-2}{G c_{s}^{2} \delta t}}\left[c_{s}^{2} \rho-\tilde{p}(\rho)\right]^{0.5}, \\
\psi^{\prime} & =\frac{d \psi}{d \rho}=\frac{1}{2} \sqrt{\frac{-2}{G c_{s}^{2} \delta t}}\left[c_{s}^{2} \rho-\tilde{p}(\rho)\right]^{-0.5}\left(c_{s}^{2}-\frac{d \tilde{p}}{d \rho}\right),
\end{aligned}
$$

and by substituting the customized EOS $\tilde{p}(\rho)$ in Eq. (23) into Eq. (25), we have

$$
\tilde{\rho}_{M}=\operatorname{root}\left[\int_{\tilde{\rho}_{V}}^{\tilde{\rho}_{L}} g_{1}\left(\rho, \tilde{\rho}_{M}\right) \frac{g_{2}\left(\rho, \tilde{\rho}_{M}\right)}{g_{3}\left(\rho, \tilde{\rho}_{M}\right)} d \rho=0\right],
$$

where

$$
\begin{aligned}
g_{1}\left(\rho, \tilde{\rho}_{M}\right)= & \tilde{p}_{0}-\tilde{p}(\rho)=\theta\left(\rho-\tilde{\rho}_{L}\right)\left(\rho-\tilde{\rho}_{V}\right)\left(\rho-\tilde{\rho}_{M}\right), \\
g_{2}\left(\rho, \tilde{\rho}_{M}\right)= & c_{s}^{2}-\frac{d \tilde{p}}{d \rho} \\
= & c_{s}^{2}-\theta\left[\left(\rho-\tilde{\rho}_{L}\right)\left(\rho-\tilde{\rho}_{V}\right)\right. \\
& \left.+\left(\rho-\tilde{\rho}_{L}\right)\left(\rho-\tilde{\rho}_{M}\right)+\left(\rho-\tilde{\rho}_{V}\right)\left(\rho-\tilde{\rho}_{M}\right)\right], \\
g_{3}\left(\rho, \tilde{\rho}_{M}\right)= & c_{s}^{2} \rho-\tilde{p}(\rho) \\
= & c_{s}^{2} \rho-\tilde{p}_{0}-\theta\left(\rho-\tilde{\rho}_{L}\right)\left(\rho-\tilde{\rho}_{M}\right)\left(\rho-\tilde{\rho}_{V}\right) .
\end{aligned}
$$

The root $\tilde{\rho}_{M}$ of this integral equation can be easily solved numerically through a few lines of code. To ensure that such a solution always exists, one should properly scale the customized EOS $\tilde{p}$ in LB units by defining $(a, b, R)$ parameters in LB units in a way that ensures that $g_{2}=c_{s}^{2}-d \tilde{p} / d \rho>0$ and $g_{3}=c_{s}^{2} \rho-\tilde{p}>0$ for any $\rho$ between $\tilde{\rho}_{V}$ and $\tilde{\rho}_{L}$ for the cubic EOS of interest. Such choices of $(a, b, R)$ in LB units make the sign of the integral function equation solely dependent on that of $g_{1}$, which leads to opposite signs of the integral function at the two limits of the $\tilde{\rho}_{M}$ spectrum $\left(\tilde{\rho}_{M}=\tilde{\rho}_{V}\right.$ and $\tilde{\rho}_{M}=\tilde{\rho}_{L}$ ). For such continuous integral function with respect to $\tilde{\rho}_{M}$, these opposite signs ensure that there will always be at least one $\tilde{\rho}_{M}$ found between $\tilde{\rho}_{V}$ and $\tilde{\rho}_{L}$ that would make the integral function go to zero.

For readers' convenience, in the Supplemental Material of this paper, we provide two sample MATLAB codes, one to calculate $\tilde{\rho}_{M}$ when $\theta$ is given by Eq. (24), and another to calculate $\tilde{\rho}_{M}$ when $\theta$ is constrained to achieve a desired surface tension [20]. For normalization purposes, we quantify the location of the middle density root in the customized EOS $\tilde{\rho}_{M}$ with respect to the values of coexisting liquid and vapor densities through the density ratio $r_{\rho}$, where

$$
r_{\rho}=\frac{\tilde{\rho}_{M}-\tilde{\rho}_{V}}{\tilde{\rho}_{L}-\tilde{\rho}_{V}}
$$

and $r_{\rho}$ values are found between zero and 1 . Values of $r_{\rho}$ close to zero indicate that $\tilde{\rho}_{M}$ is found close to vapor density values; $r_{\rho}$ values close to 1 indicate that $\tilde{\rho}_{M}$ approaches liquid density values. The values of $r_{\rho}$ when some commonly used cubic EOSs are chosen as $p_{\text {EOS }}$ in the customized EOS in Eq. (23) 
TABLE I. The value of $r_{\rho}$ for the customized EOS with some commonly used cubic EOSs.

\begin{tabular}{|c|c|c|c|c|c|c|}
\hline \multirow[b]{2}{*}{$T / T_{c}$} & \multirow[b]{2}{*}{ vdW EOS } & \multirow[b]{2}{*}{ CS EOS } & \multicolumn{2}{|c|}{ SRK EOS } & \multicolumn{2}{|c|}{ PR EOS } \\
\hline & & & $\begin{array}{c}\text { Water } \\
\omega=0.344\end{array}$ & $\begin{array}{c}\text { Methane } \\
\omega=0.0104\end{array}$ & $\begin{array}{c}\text { Water } \\
\omega=0.344\end{array}$ & $\begin{array}{c}\text { Methane } \\
\omega=0.0104\end{array}$ \\
\hline 0.95 & 0.447669 & 0.438681 & 0.423538 & 0.433240 & 0.420838 & 0.430883 \\
\hline 0.9 & 0.425956 & 0.413569 & 0.394532 & 0.406979 & 0.392025 & 0.404546 \\
\hline 0.85 & 0.409284 & 0.394690 & 0.374257 & 0.387882 & 0.372407 & 0.385814 \\
\hline 0.8 & 0.395281 & 0.379268 & 0.359033 & 0.372786 & 0.357958 & 0.371264 \\
\hline 0.75 & 0.383046 & 0.366314 & 0.347513 & 0.360526 & 0.347178 & 0.359608 \\
\hline 0.7 & 0.372153 & 0.355412 & 0.339061 & 0.350574 & 0.339340 & 0.350242 \\
\hline 0.65 & 0.362401 & 0.346411 & 0.333285 & 0.342679 & 0.333999 & 0.342857 \\
\hline 0.6 & 0.353720 & 0.339290 & 0.329810 & 0.336712 & 0.330764 & 0.337283 \\
\hline 0.55 & 0.346142 & 0.334067 & 0.328164 & 0.332573 & 0.329188 & 0.333389 \\
\hline 0.5 & 0.339769 & 0.330706 & 0.327766 & 0.330097 & 0.328748 & 0.331010 \\
\hline 0.45 & 0.334756 & 0.329012 & 0.328043 & 0.328996 & 0.328939 & 0.329885 \\
\hline 0.4 & 0.331249 & 0.328584 & 0.328582 & 0.328855 & 0.329367 & 0.329652 \\
\hline
\end{tabular}

are also listed in Table I, again for readers' convenience. These values are obtained with $a=2 / 41, b=2 / 21$, and $R=1$ for the van der Waals (vdW), Soave-Redlich-Kwong (SRK), and Peng-Robinson (PR) EOS, and $a=1, b=4$, and $R=1$ for the Carnahan-Starling (CS) EOS in LB units, as recommended by Yuan and Schaefer [6]. Unless specified otherwise, these values of $(a, b, R)$ are used throughout the paper.

For demonstration purposes, in Fig. 2, the customized EOSs [with $\theta$ defined through Eq. (24)] and the corresponding original cubic EOSs are compared at two reduced temperatures, $T_{r}=T / T_{c}=0.8$ and 0.6 . The cubic EOSs selected for comparison here are given in detail in Appendix B. By design, outside the van der Waals loop, the customized EOS always collapses with the corresponding cubic EOS. This ensures that the thermodynamically meaningful pressure-density relationship is always honored in the bulk (single-)phase regions. Inside the van der Waals loops, customized EOS loops are flattened compared to the corresponding cubic EOS since the amplitude of the customized EOS $\theta$ is chosen so the compressibility $d \tilde{p} / d \rho$ is continuous at the dew point. The flattened van der Waals loops tend to bring the extra benefit of maintaining numerical stability at large density ratios, which is further discussed shortly. It should be noted that the technique of flattening the van der Waals loop in LB scaling has been reported quite often in the literature, and has been recognized as an important strategy to enhance numerical stability of PP LB models $[5,21,22]$. In this study, the resulting smoother pressure-density dependencies within van der Waals loops were not prescribed a priori, but were arrived at as a result of the constraints placed on the EOS customization.

The customized EOS in Eq. (23) is tested in a onedimensional flat interface case with $p_{\text {EOS }}$ being the commonly used cubic EOS. The simulations are conducted in a periodic domain of $L_{x} \times L_{y}=200 \times 2$. The initial distribution of the fluid density is set as

$$
\rho(x, t=0)=\tilde{\rho}_{V}+\frac{\tilde{\rho}_{L}-\tilde{\rho}_{V}}{2}\left[\tanh \left(x_{1}\right)-\tanh \left(x_{2}\right)\right],
$$

with $x_{1}=2\left(x-L_{x} / 4\right) / W$ and $x_{2}=2\left(x-3 L_{x} / 4\right) / W$, where $W=10$ is the initial width of the two-phase interfaces. In all simulations, the effective mass $\psi$ is defined with Eq. (7), and the Shan-Chen force is computed with its original definition in Eq. (5). This Shan-Chen force is discretized with Guo's et al.'s forcing scheme [Eq. (3b)] in the LBE, which ensures the discretization has a second-order accuracy. The relaxation time $\tau$ is set to 1.25 in all cases. The values of $r_{\rho}$ used in the simulations all come from Table I.

When LB simulations reach steady state, results of twophase densities and the saturated pressure are compared with the corresponding values obtained with the Maxwell equal area rule that satisfy thermodynamic consistency. For conciseness, we define a quantity $\varepsilon$ to measure the compounded deviation of simulation results from rigorous thermodynamic consistency which requires $\rho_{L}=\tilde{\rho}_{L}, \rho_{V}=\tilde{\rho}_{V}$, and $p_{0}=\tilde{p}_{0}$. This quantity $\varepsilon$ is referred to as the error of thermodynamic inconsistency and it is defined as

$$
\varepsilon=\sqrt{\left(\frac{\rho_{L}}{\tilde{\rho}_{L}}-1\right)^{2}+\left(\frac{\rho_{V}}{\tilde{\rho}_{V}}-1\right)^{2}+\left(\frac{p_{0}}{\tilde{p}_{0}}-1\right)^{2}},
$$

where $p_{0}$ is the pressure of bulk regions obtained from the simulation. Obviously, when the simulation results of a pseudopotential LB simulation satisfy full thermodynamic consistency, $\varepsilon$ should vanish. The measured $\varepsilon$ from the simulations are plotted as functions of both the reduced temperature $T_{r}$ and the liquid-vapor phase density ratio in Fig. 3. Even with large density ratios at relatively low reduced temperatures, the deviations from thermodynamic consistency by the customized EOS are trivial and typically smaller than $0.1 \%$. In Table II, values of $\varepsilon$ generated from the current approach of customized EOSs are compared with their counterparts from the same PP LB model but using full (original) cubic EOSs. With the customized EOS, the forcing scheme by Guo et al. [Eq. (3b)] has proven fully adequate to ensure sufficient numerical stability even for cases with relatively large density ratios. However, when full cubic EOSs are used, Guo et al.'s forcing scheme is known to lead to very poor numerical stability due to the significantly underpredicted vapor phase 
(a)

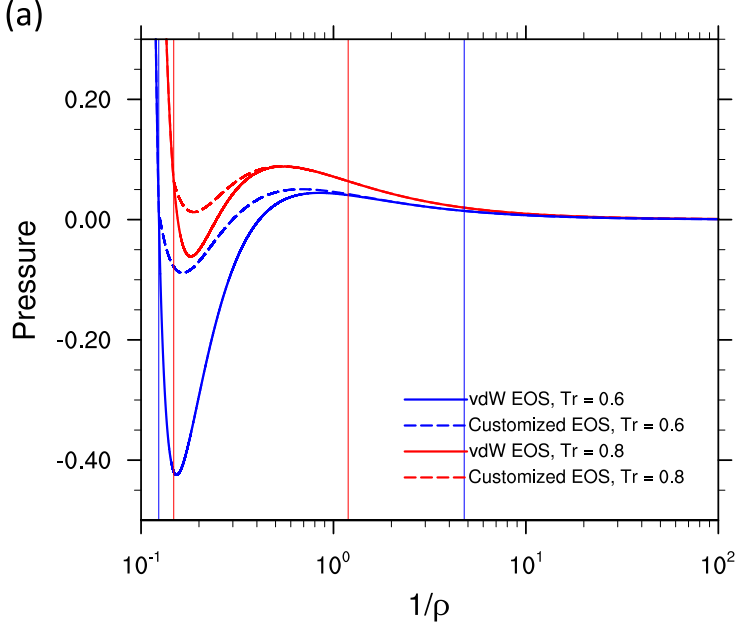

(c)

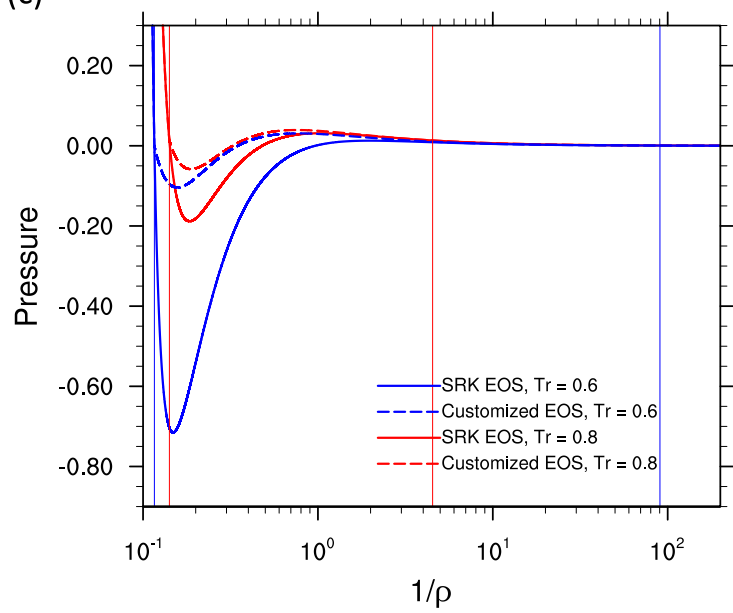

(b)

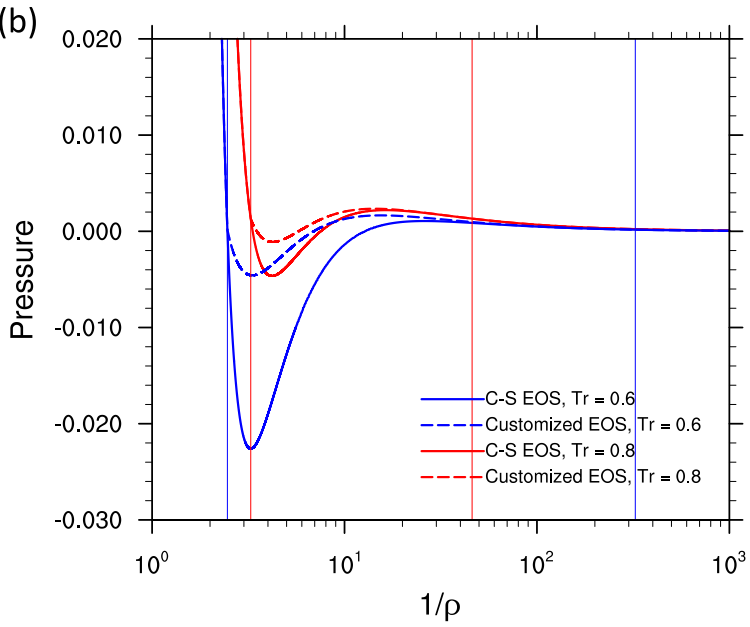

(d)

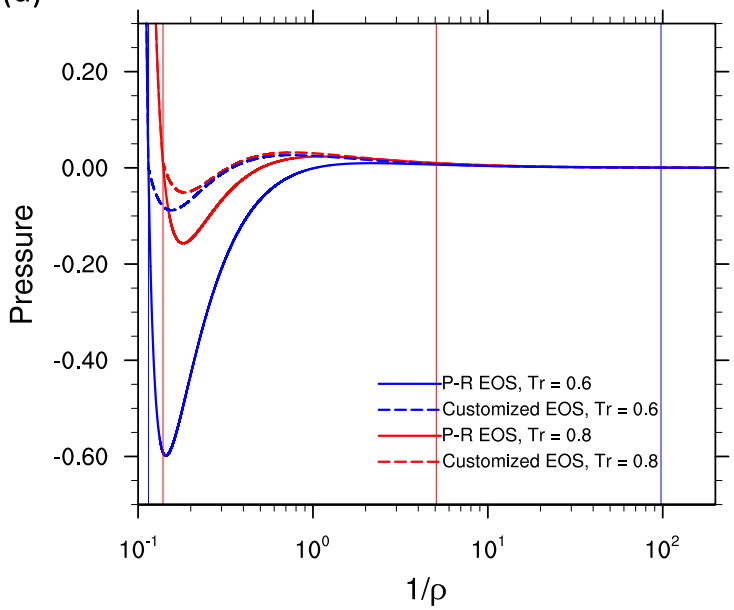

FIG. 2. A comparison between the customized EOS with the corresponding cubic EOS at two selected temperatures $T_{r}=0.8$ and $T_{r}=0.6$. $p_{\text {EOS }}$ in the customized EOS is (a) vdW EOS, (b) CS EOS, (c) SRK EOS with $\omega=0.344$ (water), and (d) PR EOS with $\omega=0.344$ (water).

densities [8]. As a remedy, the exact difference method (EDM) was proposed by Kupershtokh et al. to attain better numerical stability at higher density ratios [19]. For fair comparison, when full cubic EOSs are used, the results of $\varepsilon$ with both Guo et al.'s forcing scheme and EDM are presented in Table II. For cases where simulations are no longer stable under the current choices of parameters $(a, b, R$, and $\tau)$, results are marked as "NaN" (not a number). In Table II, it can be clearly (a)

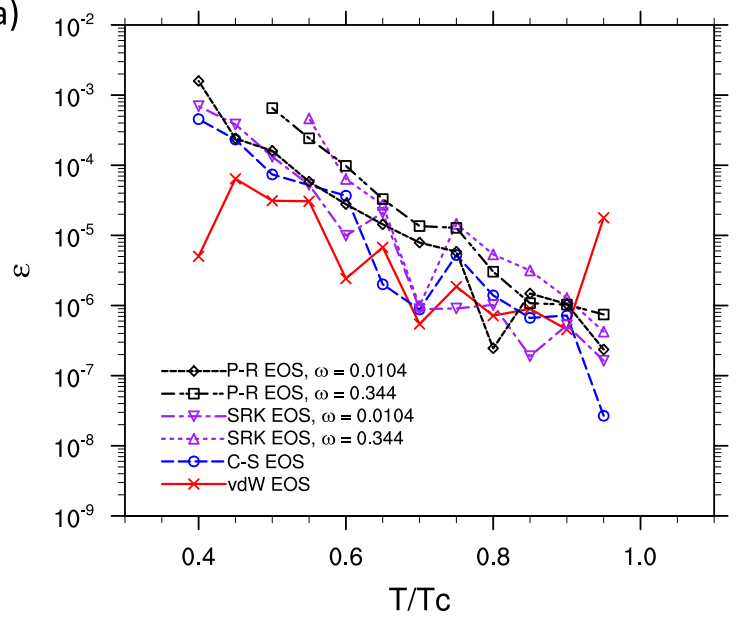

(b)

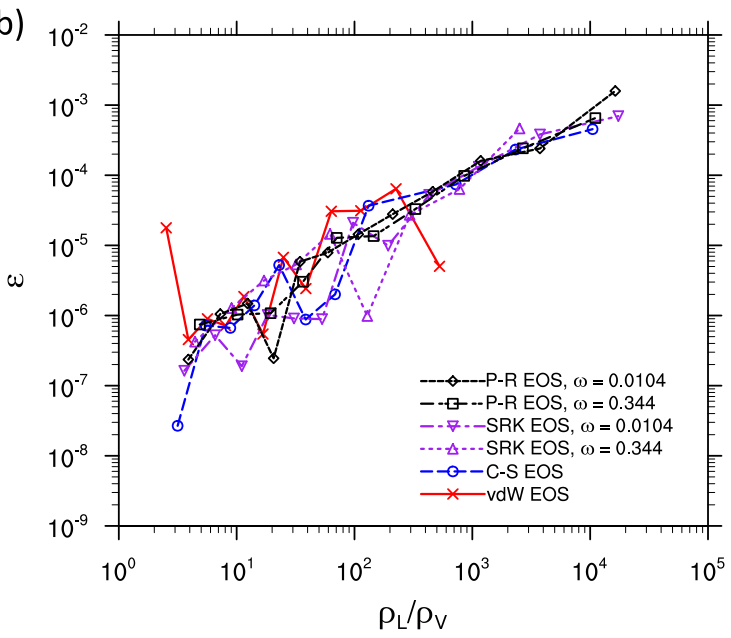

FIG. 3. The errors of thermodynamic inconsistency $\varepsilon$ as a function of (a) reduced temperature and (b) density ratio. 
TABLE II. Comparison of errors in thermodynamic inconsistency $(\varepsilon$ ) among: (a) full cubic EOS with Guo et al.'s forcing scheme, (b) full cubic EOS with EDM, and (c) customized EOS with Guo et al.'s forcing scheme (the current approach). Cases with PR and SRK EOSs use acentric factor $\omega=0.344$ only.

\begin{tabular}{lcccccccccc}
\hline \hline Case $\backslash T_{r}$ & 0.95 & 0.9 & 0.85 & 0.8 & 0.75 & 0.7 & 0.65 & 0.6 & 0.55 & 0.5 \\
\hline vdW (a) & $2.72 \times 10^{-2}$ & $8.56 \times 10^{-2}$ & $1.93 \times 10^{-1}$ & $3.77 \times 10^{-1}$ & $6.81 \times 10^{-1}$ & $1.16 \times 10^{0}$ & $\mathrm{NaN}$ & $\mathrm{NaN}$ & $\mathrm{NaN}$ & $\mathrm{NaN}$ \\
vdW (b) & $5.64 \times 10^{-3}$ & $1.79 \times 10^{-2}$ & $4.02 \times 10^{-2}$ & $7.74 \times 10^{-2}$ & $1.36 \times 10^{-1}$ & $2.271 \times 10^{-1}$ & $3.66 \times 10^{-1}$ & $5.65 \times 10^{-1}$ & $8.34 \times 10^{-1}$ & $1.12 \times 10^{0}$ \\
vdW (c) & $1.78 \times 10^{-5}$ & $4.54 \times 10^{-7}$ & $8.98 \times 10^{-7}$ & $7.15 \times 10^{-7}$ & $1.86 \times 10^{-6}$ & $5.44 \times 10^{-7}$ & $6.75 \times 10^{-6}$ & $2.42 \times 10^{-6}$ & $3.06 \times 10^{-5}$ & $3.12 \times 10^{-5}$ \\
CS (a) & $4.90 \times 10^{-2}$ & $1.68 \times 10^{-1}$ & $4.07 \times 10^{-1}$ & $8.46 \times 10^{-1}$ & $\mathrm{NaN}$ & $\mathrm{NaN}$ & $\mathrm{NaN}$ & $\mathrm{NaN}$ & $\mathrm{NaN}$ & $\mathrm{NaN}$ \\
CS (b) & $1.22 \times 10^{-2}$ & $4.07 \times 10^{-2}$ & $9.45 \times 10^{-2}$ & $1.87 \times 10^{-1}$ & $3.36 \times 10^{-1}$ & $5.61 \times 10^{-1}$ & $8.68 \times 10^{-1}$ & $1.20 \times 10^{0}$ & $1.39 \times 10^{0}$ & $1.41 \times 10^{0}$ \\
CS (c) & $2.68 \times 10^{-8}$ & $7.20 \times 10^{-7}$ & $6.65 \times 10^{-7}$ & $1.39 \times 10^{-6}$ & $5.24 \times 10^{-6}$ & $8.77 \times 10^{-7}$ & $2.00 \times 10^{-6}$ & $3.68 \times 10^{-5}$ & $4.72 \times 10^{-5}$ & $7.39 \times 10^{-5}$ \\
SRK (a) & $1.02 \times 10^{-1}$ & $3.90 \times 10^{-1}$ & $1.01 \times 10^{0}$ & $\mathrm{NaN}$ & $\mathrm{NaN}$ & $\mathrm{NaN}$ & $\mathrm{NaN}$ & $\mathrm{NaN}$ & $\mathrm{NaN}$ & $\mathrm{NaN}$ \\
SRK (b) & $2.63 \times 10^{-2}$ & $9.37 \times 10^{-2}$ & $2.25 \times 10^{-1}$ & $4.48 \times 10^{-1}$ & $7.75 \times 10^{-1}$ & $1.15 \times 10^{0}$ & $1.38 \times 10^{0}$ & $1.41 \times 10^{0}$ & $\mathrm{NaN}$ & $\mathrm{NaN}$ \\
SRK (c) & $4.24 \times 10^{-7}$ & $1.27 \times 10^{-6}$ & $3.15 \times 10^{-6}$ & $5.35 \times 10^{-6}$ & $1.46 \times 10^{-5}$ & $9.83 \times 10^{-7}$ & $2.72 \times 10^{-5}$ & $6.35 \times 10^{-5}$ & $4.64 \times 10^{-4}$ & $\mathrm{NaN}$ \\
PR (a) & $1.19 \times 10^{-1}$ & $4.47 \times 10^{-1}$ & $1.13 \times 10^{0}$ & $\mathrm{NaN}$ & $\mathrm{NaN}$ & $\mathrm{NaN}$ & $\mathrm{NaN}$ & $\mathrm{NaN}$ & $\mathrm{NaN}$ & $\mathrm{NaN}$ \\
PR (b) & $3.26 \times 10^{-2}$ & $1.13 \times 10^{-1}$ & $2.64 \times 10^{-1}$ & $5.11 \times 10^{-1}$ & $8.59 \times 10^{-1}$ & $1.23 \times 10^{0}$ & $1.41 \times 10^{0}$ & $\mathrm{NaN}$ & $\mathrm{NaN}$ & $\mathrm{NaN}$ \\
PR (c) & $7.45 \times 10^{-7}$ & $1.03 \times 10^{-6}$ & $1.08 \times 10^{-6}$ & $3.03 \times 10^{-6}$ & $1.28 \times 10^{-5}$ & $1.36 \times 10^{-5}$ & $3.29 \times 10^{-5}$ & $9.79 \times 10^{-5}$ & $2.42 \times 10^{-4}$ & $6.53 \times 10^{-4}$ \\
\hline \hline
\end{tabular}

seen that with the customized EOS, the error of thermodynamic inconsistency $\varepsilon$ can be dramatically reduced (by a few orders of magnitude) compared to the use of full cubic EOSs. Also, since the thermodynamic inconsistency is significantly reduced in the customized EOS approach, the PP LB model is able to reach much higher density ratios. It would also be desirable to contrast errors of thermodynamic inconsistency against those previous methods that have attempted to restore thermodynamic consistency in PP LB models. However, the implementation of those previous methods is limited by the difficulty of finding the associated optimal tuning parameters. For example, for Li et al.'s approach [8], a unified value of the tuning parameter $\sigma=0.105$ was given in the original reference for CS EOS at all reduced temperatures. Based on our test, this value would result in $\varepsilon=1.25 \times 10^{-1}$ at $T_{r}=$ 0.55 , which is still a few orders larger than $\varepsilon=4.72 \times 10^{-5}$ given by the current approach. To be fair, if more accurate optimal tuning parameters from those previous methods could be computed, their thermodynamic consistency could be further improved. The easiness to obtain such optimal $\tilde{\rho}_{M}$ is in fact the biggest advantage of the current approach compared to previous methods. This aspect will be further explored in Sec. V.

It is important to note that the small residual error of $\varepsilon$ by the current approach is partly due to the truncation error of $r_{\rho}$ introduced in the simulations. In Table I, all values of $r_{\rho}$ were truncated after the sixth decimal digit. When reduced temperatures are low, vapor phase density and saturated pressure will have very small values. For example, the PR EOS with $\omega=0.0104$ at $T_{r}=0.4$ leads to $\tilde{\rho}_{L}=9.270680, \tilde{\rho}_{V}=$ $5.675914 \times 10^{-4}$, and $\tilde{p}_{0}=1.653953 \times 10^{-5}$ in LB units by the present parameters of choice. At these conditions, a slight change in $r_{\rho}$ could result in notable changes in the simulation results of $\rho_{V}$ and $p_{0}$. This explains the increasing trend of $\varepsilon$ as temperature is reduced (and density ratio is increased) when the same number of significant digits is used for $r_{\rho}$, as shown in Fig. 3. To confirm, we further examined the resulting $\varepsilon$ with $r_{\rho}$ truncated at different numbers of decimal digits at the two small reduced temperatures of $T_{r}=0.5$ and
$T_{r}=0.4$. As shown in Fig. 4, $\varepsilon$ decreases as more digits are kept for $r_{\rho}$ after the decimal point, with only few exceptions. It is also observed that once the precision of $10^{-8}$ is reached, further improving $r_{\rho}$ generally does not lead to any notable improvement in $\varepsilon$. Since the precision of $r_{\rho}$ depends solely on the precision of the input parameters $\tilde{\rho}_{V}, \tilde{\rho}_{L}$, and $\tilde{p}_{0}$ when numerically solving for it, e.g., with the sample codes in the Supplemental Material [20], the readers can choose $r_{\rho}$ with any precision that leads to a satisfactory thermodynamic consistency needed for their applications.

It is worth noting that, regardless of the users' choices of cubic EOS for $p_{\mathrm{EOS}}, r_{\rho}$ roughly follows the same generalized trend with respect to liquid-vapor density ratios. For readers to see this trend, $r_{\rho}$ values for all cases are compiled together as a function of density ratio in Fig. 5. As per the preceding discussion, $r_{\rho}$ needs to have sufficient precision to ensure full thermodynamic consistency in PP LB simulations. Readers are recommended to use the sample codes in the Supplemental Material [20] or their own codes to compute $r_{\rho}$ with the precision that meets their needs. Because the $r_{\rho}$ values can be easily obtained at any desired precision, $r_{\rho}$ should not be derived from any generalized empirical function that could be fit from Fig. 5; otherwise, it will likely fail the precision requirement. We therefore do not include any mathematical expression for the empirical function that appears in Fig. 5.

As mentioned earlier in this section, one extra benefit of the current approach is that the customized EOSs typically have flattened van der Waals loops compared to the corresponding cubic EOSs, which enhance the numerical stability of PP LB models for large density ratios. In Table III, the maximum density ratios achieved by the current approach in the above flat interface case are listed for different $p_{\mathrm{EOS}}$. The profiles of the normalized density at the largest density ratio achieved by each case are also shown in Fig. 6. The thickness of the interface, measured by the region with $1.1 \rho_{V} \leqslant \rho \leqslant 0.9 \rho_{L}$ ranges from $11 \delta x$ at the density ratio of $\rho_{L} / \rho_{V} \approx 790$ to $18 \delta x$ at the density ratio around 33000 , which is acceptably small. Even larger density ratios can be achieved by reducing the value of $a$ in cubic EOSs in LB units, with the price of further sacrificing the interface sharpness [5]. 

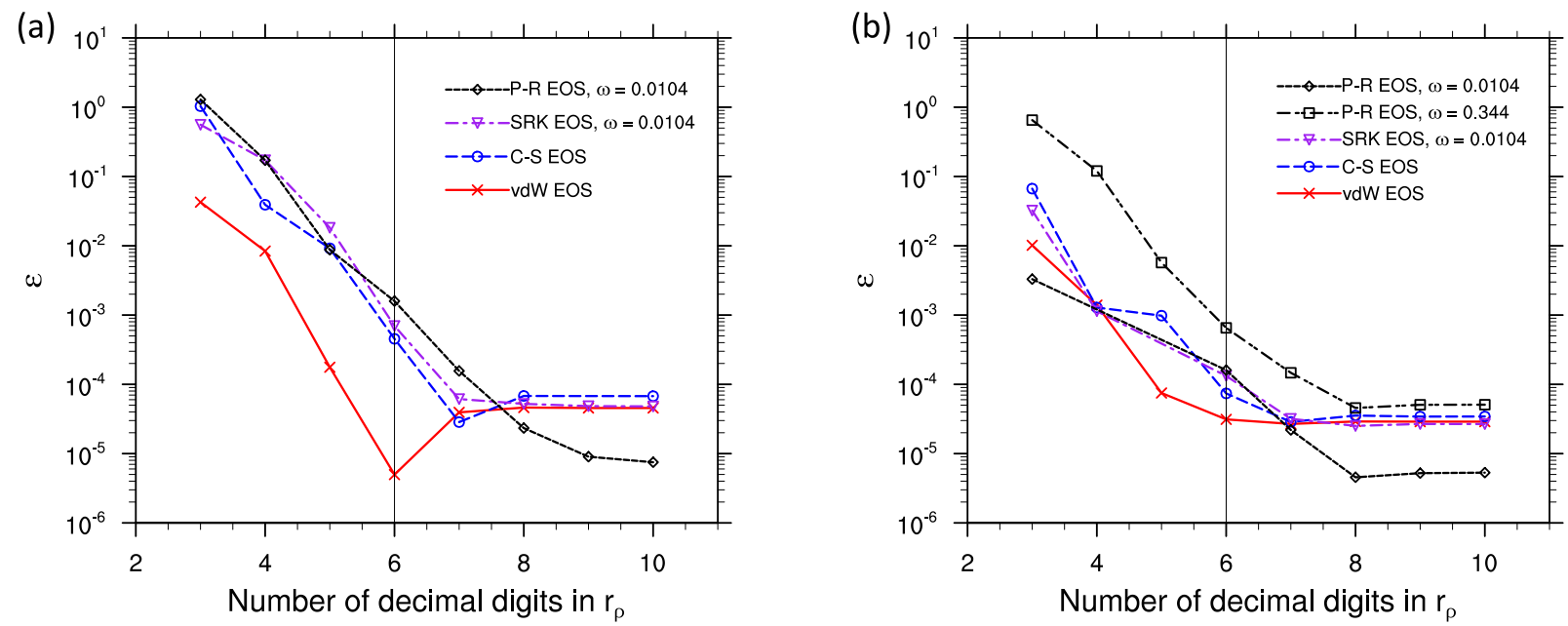

FIG. 4. The errors of thermodynamic inconsistency $\varepsilon$ as a function of the precision of $r_{\rho}$ at (a) $T_{r}=0.4$ and (b) $T_{r}=0.5$. Cases of P-R EOS and SRK EOS with $\omega=0.344$ are not numerically stable under current choices of parameters $(a, b, R$, and $\tau)$ at $T_{r}=0.4$. The case of SRK EOS with $\omega=0.344$ is not numerically stable under current choices of parameters at $T_{r}=0.5$.

\section{COMPARISON AMONG DIFFERENT WAYS OF IMPROVING THE THERMODYNAMIC CONSISTENCY OF PP LB MODELS AND DISCUSSION}

Similar to the previous efforts of improving the thermodynamic consistency in PP LB models [8,14,19], the approach proposed in this study also introduces Shan-Chen force calibration parameters but with a different perspective: through a customized EOS. The biggest advantage of the proposed approach is that finding the appropriate values for those calibration parameters does not need any time-consuming trialand-error approach that would require conducting multiple LB simulations, but rather they can be obtained beforehand via simple codes in seconds. Those values are also sufficiently accurate to ensure the largest error in the simulation results of coexisting density and saturated pressure to be as low as

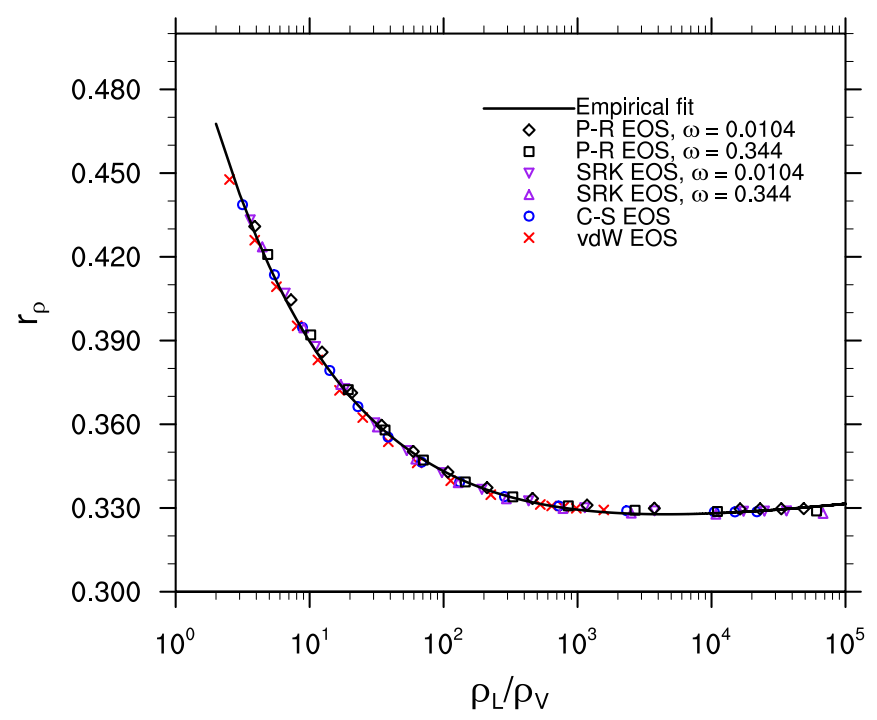

FIG. 5. The values of $r_{\rho}$ as a function of density ratio for different choices of $p_{\mathrm{EOS}}$.
$0.01 \%$, even at a density ratio up to $10^{4}$, which has never been reported by previous efforts.

At this point, readers may wonder if the optimal values of the tuning parameters introduced in those previous efforts, e.g., Refs. $[8,14,19]$, could be found in the same way through solving the integral mechanical stability condition, which avoids trial and error. Indeed, following the derivation in Appendix A, we are able to derive the mechanical stability conditions for these previous efforts. For example, both $\mathrm{Ku}-$ pershtokh et al.'s approach [19] and Li et al.'s approach [8] would result in the same mechanical stability condition that reads

$$
\int_{\tilde{\rho}_{V}}^{\tilde{\rho}_{L}}\left(\tilde{p}_{0}-p_{\mathrm{EOS}}\right) \psi^{x} \psi^{\prime} d \rho=0,
$$

where $x=1-2 \beta$ for Kupershtokh et al.'s approach and $x=$ $16 G \sigma-1$ for Li et al.'s approach. When one chooses $\beta=1$ in Kupershtokh et al.'s definition of Shan-Chen force and $\sigma=0$ in $\mathrm{Li}$ et al.'s forcing scheme, the original definition of the Shan-Chen force and Guo et al.'s forcing scheme are recovered, and the above mechanical stability condition reverts back to Eq. (8).

Kharmiani et al.'s model [14], on the other hand, does not produce a mechanical stability condition that takes into consideration the discrete nature of the LBM. In other words, this model does not result in a mechanical stability condition following Shan's derivation [10] or the derivation provided in Appendix A. However, it can still produce a mechanical stability condition if He and Doolen's derivation [9] is followed, which turns out to be Eq. (32) once again but with $x=-2-\lambda$. As pointed out by Shan [10], He and Doolen's derivation fails to consider the discrete nature of LBM simulations, so even if an optimal $\lambda$ is solved from Eq. (32), it would not be able to enforce full thermodynamic consistency with Kharmiani et al.'s model in actual simulations. We therefore exclude this model from the discussion.

By inputting $p_{\text {EOS }}$ and the corresponding $\tilde{\rho}_{V}, \tilde{\rho}_{L}$, and $\tilde{p}_{0}$ of the cubic EOS of choice, values of " $x$ " satisfying Eq. (32) 
TABLE III. The maximum density ratios achieved by the customized EOS. $a=2 / 49, b=2 / 21$, and $R=1$ in the LB units are used with vdW EOS, SRK EOS, and PR EOS; $a=1, b=4$, and $R=1$ in the LB units are used with CS EOS.

\begin{tabular}{|c|c|c|c|c|c|c|}
\hline & \multicolumn{2}{|c|}{ PR EOS } & \multicolumn{2}{|c|}{ SRK EOS } & \multirow[b]{2}{*}{ vdW EOS } & \multirow[b]{2}{*}{ CS EOS } \\
\hline & $\omega=0.344$ & $\omega=0.0104$ & $\omega=0.344$ & $\omega=0.0104$ & & \\
\hline$\tilde{\rho}_{L} / \tilde{\rho}_{V}$ & $\begin{array}{c}\sim 11000 \\
\left(T_{r}=0.50\right)\end{array}$ & $\begin{array}{c}\sim 33000 \\
\left(T_{r}=0.38\right)\end{array}$ & $\begin{array}{c}\sim 7800 \\
\left(T_{r}=0.51\right)\end{array}$ & $\begin{array}{c}\sim 17000 \\
\left(T_{r}=0.40\right)\end{array}$ & $\begin{array}{c}\sim 790 \\
\left(T_{r}=0.38\right)\end{array}$ & $\begin{array}{c}\sim 15000 \\
\left(T_{r}=0.39\right)\end{array}$ \\
\hline
\end{tabular}

could be solved. For example, for the CS EOS with $a=$ $1, b=4$, and $R=1$ at a temperature $T_{r}=0.6$, we have $x=-2.76226638$, which corresponds to $\beta=1.88113319$ or $\sigma=0.11014165$. However, even with those values, precise thermodynamic consistency is still not achieved in actual simulations. In the test case shown in Sec. IV, with $\beta=$ 1.88113319 we get $\rho_{L}=0.40641$ and $\rho_{V}=5.670643 \times 10^{-3}$ by Kupershtokh et al.'s approach, and with $\sigma=0.11014165$, we get $\rho_{L}=0.40624$ and $\rho_{V}=3.62279 \times 10^{-3}$ by Li et al.'s forcing scheme; both deviate significantly from the physical values $\tilde{\rho}_{L}=0.40619$ and $\tilde{\rho}_{V}=3.08242 \times 10^{-3}$ predicted by the Maxwell equal area rule. These deviations were unexpected since there seems to be no clear reason for them. A reasonable guess would be that the nonlinear parts introduced by those approaches, i.e., the second term in Eq. (13) and the last part of Eq. (18) creating discrepancies between the integral mechanical stability condition Eq. (32), from which " $x$ " is computed, and actual LB simulations based on discrete points. For the integral mechanical stability condition to be fully honored by LB simulations, both the original definition of the Shan-Chen force and Guo et al.'s forcing scheme are likely required. These observations inspired us to retain the original definition of the Shan-Chen force and Guo et al.'s forcing scheme and rather seek an alternative way to introduce calibration at the EOS level. That perspective eventually led to the EOS customization approach presented in this paper.

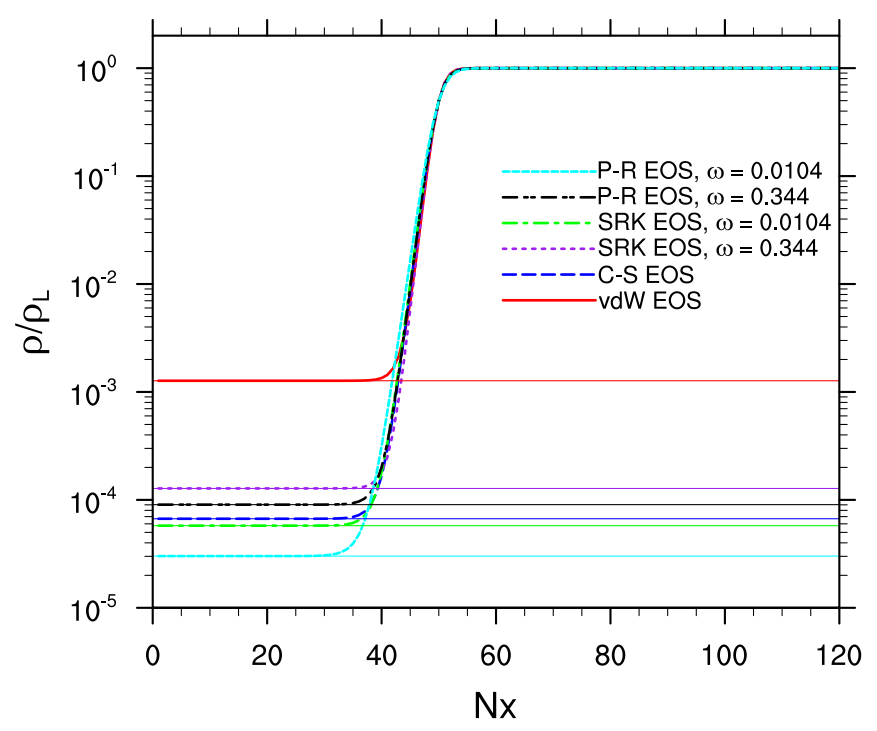

FIG. 6. The profiles of the fluid density at the maximum density ratio achieved by the customized EOS with different choices of $p_{\text {EOS }}$. Each thin horizontal line with the same color represents the vapor phase density in each case predicted by the Maxwell equal area rule.

\section{ACHIEVING DESIRED SURFACE TENSION VIA THE CUSTOMIZED EOS}

As demonstrated in Fig. 2, the proposed approach of EOS customization is likely to affect interface physics since it can lead to flattened van der Waals loops compared to those predicted by the original cubic EOS. While it has been discussed that flattening the van der Waals loops is recognized as a key strategy to enhance the numerical stability of PP LB models (see, e.g., Refs. [5,21,22]), the effect of the proposed EOS customization on interface surface tension needs to be further discussed.

Interface physics in a PP LB model is often examined via its resulting pressure tension. Since the current approach does not change the definition of the Shan-Chen force or the forcing scheme in the LBM, the resulting pressure tensor remains identical to that of the original PP LB model, i.e., the one presented by Shan [10] with the nearest-neighbor interactions

$$
\begin{aligned}
P_{i j}= & \delta_{i j}\left[c_{s}^{2} \rho+\frac{1}{2} \delta t G c_{s}^{2} \psi^{2}+\frac{3}{4} \delta t^{3} G c_{s}^{4}\left(\psi \nabla^{2} \psi\right)\right. \\
& \left.+\frac{1}{2} \delta t^{3} G c_{s}^{4}(\nabla \psi \cdot \nabla \psi)\right]-\frac{1}{2} \delta t^{3} G c_{s}^{4} \partial_{i} \psi \partial_{j} \psi
\end{aligned}
$$

This represents a key difference with respect to previous approaches, which rely on modifying the above pressure tensor in different ways to restore thermodynamic consistency in PP LB models $[8,14]$. In the current approach, the calculation of $\psi$ is still through Eq. (7), but $\psi$ has been modified through the customized EOS rather than the full cubic EOSs adopted in many other PP LB models.

By definition (e.g., Refs. $[9,10]$ ), the surface tension $\gamma$ attained in pseudopotential LB models can be quantified as

$$
\gamma=\int_{-\infty}^{+\infty}\left(P_{x x}-P_{y y}\right) d x
$$

where $P_{x x}$ and $P_{y y}$ are normal and transverse components of the pressure tensor. Assuming $\psi$ is only a function of $x$, and substituting the pressure tensor in Eq. (33) into the surface tension definition above, we have

$$
\begin{aligned}
\gamma & =-\frac{1}{2} G \delta t^{3} c_{s}^{4} \int_{-\infty}^{+\infty}\left|\frac{d \psi}{d x}\right|^{2} d x \\
& =-\frac{1}{2} G \delta t^{3} c_{s}^{4} \int_{\rho_{V}}^{\rho_{L}} \psi^{\prime 2} \sqrt{z(\rho)} d \rho
\end{aligned}
$$

where

$$
z(\rho)=\frac{8}{3 \delta t^{3} G c_{s}^{4} \psi^{\prime 2}} \int_{\rho_{V}}^{\rho}\left[p_{0}-p_{\mathrm{EOS}}(\hat{\rho})\right] \frac{\psi^{\prime}}{\psi} d \hat{\rho} .
$$


TABLE IV. Theoretical surface tension coefficients with the customized EOSs [e.g., vdW(c)] and their corresponding cubic EOSs (e.g., vdW). Results from the SRK EOS and PR EOS are obtained with $\omega=0.344$ (water).

\begin{tabular}{|c|c|c|c|c|c|c|c|c|}
\hline$T_{r}$ & $\operatorname{vdW}(\mathrm{c})$ & $\mathrm{vdW}$ & $\mathrm{CS}(\mathrm{c})$ & $\mathrm{CS}$ & $\mathrm{PR}(\mathrm{c})$ & PR & SRK(c) & SRK \\
\hline 0.9 & $2.027 \times 10^{-2}$ & $2.099 \times 10^{-2}$ & $1.082 \times 10^{-3}$ & $1.092 \times 10^{-3}$ & $3.555 \times 10^{-2}$ & $3.511 \times 10^{-2}$ & $3.714 \times 10^{-2}$ & $3.718 \times 10^{-2}$ \\
\hline 0.8 & $5.064 \times 10^{-2}$ & $5.373 \times 10^{-2}$ & $2.729 \times 10^{-3}$ & $2.884 \times 10^{-3}$ & $7.814 \times 10^{-2}$ & $8.407 \times 10^{-2}$ & $8.287 \times 10^{-2}$ & $8.931 \times 10^{-1}$ \\
\hline 0.7 & $8.210 \times 10^{-2}$ & $8.910 \times 10^{-2}$ & $4.414 \times 10^{-3}$ & $4.973 \times 10^{-3}$ & $1.088 \times 10^{-1}$ & $1.330 \times 10^{-1}$ & $8.931 \times 10^{-2}$ & $1.411 \times 10^{-1}$ \\
\hline 0.6 & $1.099 \times 10^{-1}$ & $1.234 \times 10^{-1}$ & $5.807 \times 10^{-3}$ & $7.234 \times 10^{-3}$ & $1.227 \times 10^{-1}$ & $1.790 \times 10^{-1}$ & $1.329 \times 10^{-1}$ & $1.892 \times 10^{-1}$ \\
\hline 0.5 & $1.294 \times 10^{-1}$ & $1.551 \times 10^{-1}$ & $6.636 \times 10^{-3}$ & $9.652 \times 10^{-3}$ & $1.225 \times 10^{-1}$ & $2.214 \times 10^{-1}$ & $1.331 \times 10^{-1}$ & $2.331 \times 10^{-1}$ \\
\hline
\end{tabular}

Unfortunately, when cubic EOSs are used, the above definition of theoretical surface tension often breaks down and fails to generate a meaningful result. This is because, for the theoretical surface tension to be thermodynamically meaningful, $p_{0}, \rho_{V}$, and $\rho_{L}$ in Eqs. (35) and (36) should be always replaced by their physical values, $\tilde{p}_{0}, \tilde{\rho}_{V}$, and $\tilde{\rho}_{L}$, respectively. However, since the use of full cubic EOSs in the PP LB model does not guarantee thermodynamic consistency, $z(\rho)$ could have negative values, which leads to complex values of the theoretical surface tension. On the other hand, if $p_{0}$, $\rho_{V}$, and $\rho_{L}$ are assigned with the actual values predicted by the PP LB model with full cubic EOSs, although it ensures a real value of theoretical surface tension is obtained, this value is not endorsed by thermodynamics. To resolve this conflict, while $p_{0}, \rho_{V}$, and $\rho_{L}$ in Eqs. (35) and (36) are still replaced by their corresponding physical values, $z(\rho)$ under the square root is also replaced by its absolute value $|z(\rho)|$. This special treatment is only necessary when computing theoretical surface tensions for full cubic EOSs, as the customized EOS always satisfies thermodynamic consistency. With this precaution, the resulting theoretical surface tensions with the customized EOS and its corresponding cubic EOS are tabulated in Table IV in LB units. Compared with the cubic EOS, the surface tension coefficients of the customized EOS match well with their corresponding values from the cubic EOS at large reduced temperatures, but can be underpredicted at lower reduced temperatures.

From Table IV, particularly for low reduced temperatures, it may appear that the proposed EOS customization may be trading accuracy in interface physics (in terms of surface tension) in exchange for enhanced thermodynamic consistency in the bulk phase regions. This does not have to be the case. Surface tension can be straightforwardly adjusted using the customized EOS via the adjustment of the value of $\theta$, which controls the amplitude of the customized van der Waals loop. Adjustable surface tension is a desirable numerical feature that had been pursued in quite a few PP LB models $[11,14]$. Such flexibility is desired by PP LB models in order to further increase their adaptivity to tackle realistic fluid flow problems rather than being fully consistent with thermodynamic behavior (as surface tension is physically nonadjustable with a given fluid at a certain temperature) [9].

In our proposed EOS customization, thermodynamic consistency is ensured by finding an appropriate middle-root $\tilde{\rho}_{M}$ for the customization. Such an optimal $\tilde{\rho}_{M}$ would adjust itself following any $\theta$ selected by the user of the approach. In Sec. IV, we selected $\theta$ as the one prescribed by Eq. (24), which ensured compressibility $d p_{\mathrm{EOS}} / d \rho$ continuity at the dew point $\rho=\tilde{\rho}_{V}$. This was the $\theta$ choice used to generate Table IV. However, since $\theta$ is not essential in achieving thermodynamic consistency, it can be made a free parameter to achieve the desired surface tension. For convenience, $\theta$ can be best quantified by introducing another nondimensional parameter $r_{\theta}$ defined as

$$
\begin{aligned}
\theta= & \left.\frac{\left(1-r_{\theta}\right)}{\left(\tilde{\rho}_{V}-\tilde{\rho}_{M}\right)\left(\tilde{\rho}_{V}-\tilde{\rho}_{L}\right)} \frac{d p_{\mathrm{EOS}}}{d \rho}\right|_{\rho=\tilde{\rho}_{V}} \\
& +\left.\frac{r_{\theta}}{\left(\tilde{\rho}_{L}-\tilde{\rho}_{M}\right)\left(\tilde{\rho}_{L}-\tilde{\rho}_{M}\right)} \frac{d p_{\mathrm{EOS}}}{d \rho}\right|_{\rho=\tilde{\rho}_{L}} .
\end{aligned}
$$

When $r_{\theta}=1$, the compressibility $d p_{\mathrm{EOS}} / d \rho$ is continuous at the bubble point; when $r_{\theta}=0$, the compressibility $d p_{\mathrm{EOS}} / d \rho$ is continuous at the dew point, which takes the $\theta$ customization back the one proposed in Eq. (24). In order to further examine and quantify the effect of $r_{\theta}$ selection on surface tension, Table $\mathrm{V}$ shows how theoretical surface tension values change with changing $r_{\theta}$ for the customized EOS, for the cases of vdW and PR EOS and two reduced temperatures $\left(T_{r}=\right.$ 0.8 and $T_{r}=0.6$ ). It is clear that proper adjustments of $r_{\theta}$ could match the surface tension predicted by the full original cubic EOS.

To confirm that the thermodynamic consistency would not be affected by varying $r_{\theta}$ in the customized EOS, in Fig. 7 we plot the density profiles at the steady state of the flat interface case (same case as in Sec. IV) with different $r_{\theta}$ for the two cases at $T_{r}=0.6$ in Table $\mathrm{V}$. It can be seen that, regardless the choice of $r_{\theta}$, full thermodynamic consistency can always be achieved by the customized EOS. However, varying $r_{\theta}$ does allow us to change the thickness of the interface and the resulting surface tension. The larger $r_{\theta}$ is, the steeper the customized van der Waals loop would be, which leads to larger surface tension due to steeper density gradients at the interface and thinner interface thicknesses. At the same time, large $r_{\theta}$ could offset the benefit of achieving better numerical stability in PP LB models for large density ratios by flattening the van der Waals loops, as discussed in Sec. IV. Readers are given the freedom to determine the proper selection of customized EOS parameters that meets their specific needs.

\section{CONCLUSIONS AND FINAL REMARKS}

In this document, we discussed the inadequacies found in previous efforts that attempted to restore thermodynamic consistency in PP LB models. The ultimate goal was defined as finding a solution that can achieve rigorous thermodynamic 
TABLE V. Theoretical surface tension of the customized EOS with different values of $r_{\theta}$. The last column shows the theoretical surface tension generated by the full cubic EOS and the value of $r_{\theta}$ reproduces this value by the corresponding customized EOS. The unavailable data means the resulting surface tension is nonphysical (zero or negative).

\begin{tabular}{lccccc}
\hline \hline & $r_{\theta}=0$ & $r_{\theta}=-0.025$ & $r_{\theta}=0.025$ & $r_{\theta}=0.05$ & Full EOS \\
\hline$T_{r}=0.8, \mathrm{vdW}(\mathrm{c})$ & $5.064 \times 10^{-2}$ & $4.917 \times 10^{-2}$ & $5.206 \times 10^{-2}$ & $5.346 \times 10^{-2}$ & $5.373 \times 10^{-2}\left(r_{\theta}=0.0550\right)$ \\
$T_{r}=0.8, \mathrm{PR}(\mathrm{c})$ & $7.814 \times 10^{-2}$ & $7.552 \times 10^{-2}$ & $8.061 \times 10^{-2}$ & $8.309 \times 10^{-2}$ & $8.407 \times 10^{-1}\left(r_{\theta}=0.0609\right)$ \\
$T_{r}=0.6, \mathrm{vdW}(\mathrm{c})$ & $1.099 \times 10^{-1}$ & $1.008 \times 10^{-1}$ & $1.184 \times 10^{-1}$ & $1.264 \times 10^{-1}$ & $1.234 \times 10^{-1}\left(r_{\theta}=0.0405\right)$ \\
$T_{r}=0.6, \mathrm{PR}(\mathrm{c})$ & $1.227 \times 10^{-1}$ & & $1.399 \times 10^{-1}$ & $1.556 \times 10^{-1}$ & $1.790 \times 10^{-1}\left(r_{\theta}=0.0914\right)$ \\
\hline \hline
\end{tabular}

consistency, i.e., negligible deviations in the LB simulation results of coexisting densities with respect to true thermodynamic values, but without any necessity of empirical tuning. To fulfill this goal, a simple cubic-shaped, two-parameter customized EOS was introduced to replace the van der Waals loops of cubic EOSs during the calculation of effective mass in PP LB models. The customization was restricted to the van der Waals loops only, so thermodynamically meaningful pressure-density relationships in single-phase bulk regions could be maintained.

Compared to the previous efforts, the most significant advantage of the proposed approach is that the two associated customization parameters can be analytically, easily, and reliably solved through the mechanical stability condition; i.e., tuning is not needed. In particular, the parameter $\theta$ in the customized EOS is determined to obtain the desired surface tension, while the other parameter $\tilde{\rho_{M}}$ is found to ensure the full thermodynamic consistency is achieved. With the customized EOS, the pseudopotential LB model is not only able to suppress the numerical errors of coexisting density and saturated pressure to less than $0.01 \%$ for liquid-vapor density ratios ranging from $O(1)$ to $O\left(10^{4}\right)$, but it is also able to realize desired values of surface tension. For readers' convenience, sample MATLAB codes of finding the optimal parameters in the customized EOS were also provided. From the users' point of view, the proposed approach offers a complete solution for the problem of thermodynamic inconsistency in PP LB models for liquid-vapor transitions in flat interfaces.

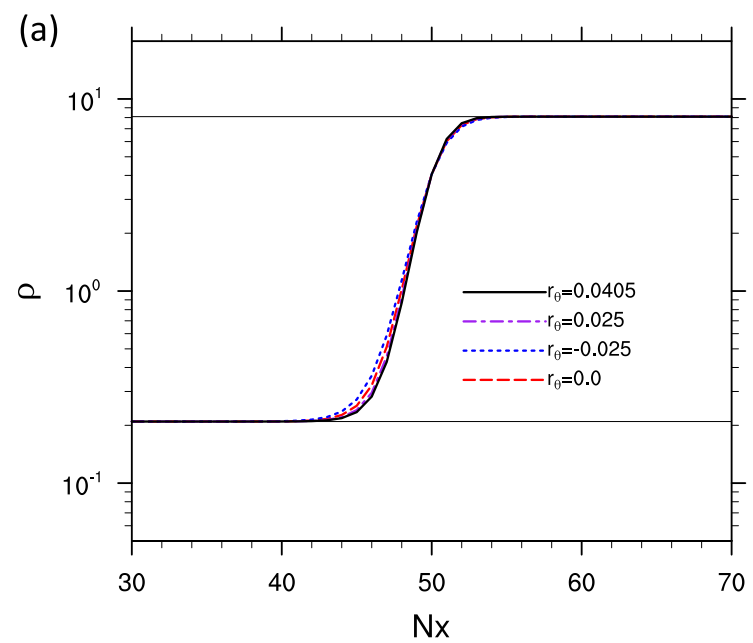

\section{ACKNOWLEDGMENTS}

Funding support from Energi Simulation and the William A. Fustos Family Professorship in Energy and Mineral Engineering at Penn State University is gratefully acknowledged.

\section{APPENDIX A: DERIVATION OF THE DISCRETE AND INTEGRAL FORMS OF THE MECHANICAL STABILITY CONDITION}

In this Appendix, we provide a detailed derivation of the integral and discrete forms of the mechanical stability condition in PP LB models, i.e., Eqs. (8) and (12). We again emphasize that both conditions are derived based on the original definition of the Shan-Chen force, i.e., Eq. (5), and Guo et al.'s forcing scheme, i.e., Eq. (3b). The mechanical stability conditions associated with those previous efforts to improve the thermodynamic consistency in PP LB models, e.g., Kupershtokh et al.'s modified definition of the ShanChen force or Li et al.'s modified forcing scheme, can be obtained following the same derivation, whose final results are also provided.

Consider a two-phase system separated by a onedimensional flat interface covering a finite number of grid points. As shown in Fig. 8, the grid points on the left of $j=1$ are in the single-phase liquid region, the grid points on the right of $j=n$ are in the single-phase vapor region, and the grid points with indices $1 \leqslant j \leqslant n$ are in the interface. At the steady state, the system is at its equilibrium. There is no net

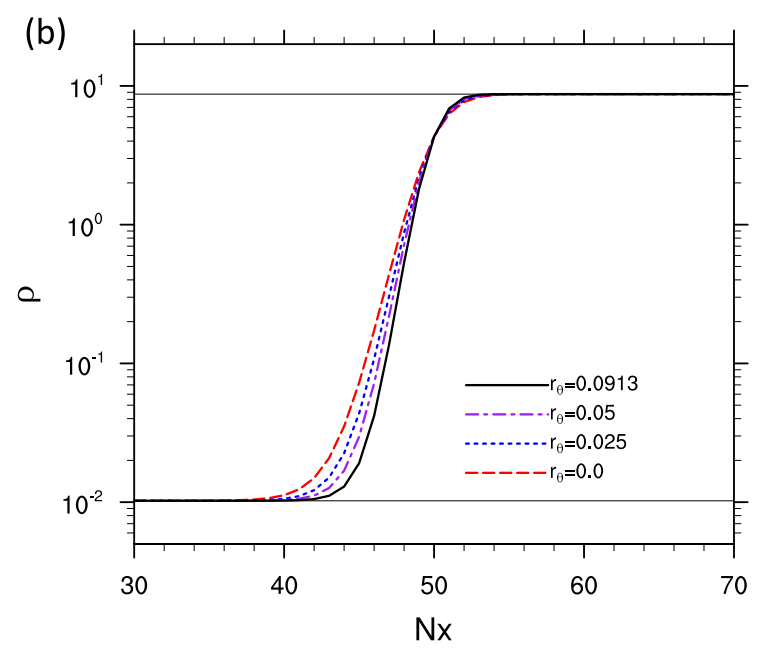

FIG. 7. The profiles of the fluid density with different $r_{\theta}$ in the customized EOS: (a) vdW EOS at $T_{r}=0.6$ and (b) PR EOS at $T_{r}=0.6$. 


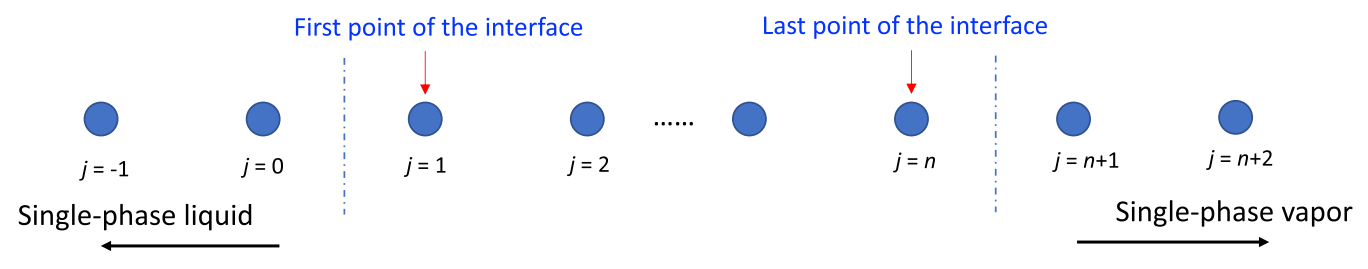

FIG. 8. Sketch of a finite-thickness 1D flat interface generated in a PP LB simulation.

mass exchange between any neighboring grid points; thus, the fluid velocity is zero everywhere in the system.

The above system can be described with a PP LB model on a D1Q3 lattice grid, which contains only three discrete velocities: $e_{0}=0, e_{-1}=-1$, and $e_{1}=1$. Two-dimensional and three-dimensional lattices with more discrete velocities can be projected onto this D1Q3 lattice for the same onedimensional case. The three distribution functions are $f_{0}, f_{1}$, and $f_{-1}$, corresponding to the discrete velocities with the same subscripts. The weighting factors $w_{\alpha}$ in the equilibrium distribution functions and forcing terms, i.e., Eq. (3), are $w_{0}=2 / 3$ and $w_{1}=w_{-1}=1 / 6$. The value of the speed of sound is $c_{s}=\sqrt{1 / 3}$.

At the steady state, the distribution functions are no longer time dependent. With zero velocity everywhere in the system, the equilibrium distribution functions and the forcing terms can be simplified as

$$
\begin{aligned}
f_{0}^{(e q)}(j) & =\frac{2}{3} \rho(j), \quad f_{1}^{(e q)}(j)=\frac{1}{6} \rho(j), \quad f_{-1}^{(e q)}(j)=\frac{1}{6} \rho(j), \\
F_{0}(j) & =0, \quad F_{1}(j)=\left(\frac{1}{2}-\frac{1}{4 \tau}\right) F(j) \delta t, \\
F_{-1}(j) & =-\left(\frac{1}{2}-\frac{1}{4 \tau}\right) F(j) \delta t,
\end{aligned}
$$

where $\rho(j)$ and $F(j)$ are the fluid density and Shan-Chen force at the grid point $j$, respectively. For the staying distribution function $f_{0}$, the LBE [Eq. (1)] becomes

$$
f_{0}(j)-f_{0}(j)=-\frac{1}{\tau}\left[f_{0}(j)-f_{0}^{(e q)}(j)\right]+F_{0}(j),
$$

which leads to $f_{0}(j)=2 / 3 \rho(j)$. Then, according to the relationships between the hydrodynamic quantities and the distribution functions [Eq. (4)], we can expressed the other two distribution functions $f_{1}$ and $f_{-1}$ as

$$
f_{-1}(j)=\frac{1}{6} \rho(j)+\frac{1}{4} F(j) \delta t, \quad f_{1}(j)=\frac{1}{6} \rho(j)-\frac{1}{4} F(j) \delta t .
$$

Substituting these expressions of $f_{1}$ and $f_{-1}$ into the LBE, we end up with

$$
\begin{aligned}
& \frac{1}{6} \rho(j-1)+\frac{1}{4} F(j-1) \delta t=\frac{1}{6} \rho(j)-\frac{1}{4} F(j) \delta t, \\
& \frac{1}{6} \rho(j+1)-\frac{1}{4} F(j+1) \delta t=\frac{1}{6} \rho(j)+\frac{1}{4} F(j) \delta t .
\end{aligned}
$$

These two equations are essentially equivalent. Regardless of how the local Shan-Chen force $F(j)$ is defined, Eqs. (A4) are always satisfied by the LB simulations if the equilibrium distribution functions and forcing terms in Eqs. (3) are used. Any mechanical stability condition followed by the LB simulations should be able to be derived from Eqs. (A4). In particular, substituting the original definition of the Shan-Chen force in Eq. (5) into the first of Eqs. (A4) would result in

$$
\begin{aligned}
& \frac{1}{6}[\rho(j-1)-\rho(j)] \\
& \quad=\frac{1}{24} G \delta t[\psi(j) \psi(j+1)-\psi(j-1) \psi(j-2)] .
\end{aligned}
$$

An important implication from Eq. (A5) is that, even inside the single-phase bulk regions, the fluid density is not precisely constant; i.e., we should not expect precisely $\rho(j)=\rho_{L}$ for all $j<1$ or $\rho(j)=\rho_{V}$ for all $j>n+1$. This is because if we have $\rho(-1)=\rho(0)=\rho_{L}$ and $\psi(-2)=$ $\psi(-1)=\psi(0)=\psi\left(\rho_{L}\right)$, Eq. (A5) will force $\psi(1)=\psi\left(\rho_{L}\right)$ or $\rho(1)=\rho_{L}$, which would recursively lead to all node points having the identical density $\rho_{L}$. This clearly violates the twophase scenario in the assumption. In reality, within the singlephase bulk regions, the fluid densities on two adjacent node points will have a negligible, but nonzero variation. This tiny variation accumulates and, due to the nonlinearity of Eq. (A5), it eventually forms a finite-thickness interface over which the fluid density changes rapidly from one phase to the other. The amplitude of the van der Waals loop in the LB units plays an important role in determining how fast the fluid density transition would happen and how thick the formed interface would be. Since the customized EOS in Sec. IV reduces the amplitude of the van der Waals loops compared to the corresponding cubic EOSs, it results in a thicker interface that benefits the numerical stability, especially when the liquidvapor density ratios are large. For the same reason, when a full cubic EOS is used, one can create a thicker interface by reducing its $a \propto R^{2} T_{c}^{2} / P_{c}$ in the LB units. Some previous studies used this technique to achieve large density ratios in their PP LB simulations, but often traded off with very thick interfaces that are not suitable for practical uses $[13,19,23]$.

Summing Eq. (A5) up over the interface, e.g., from $j=$ 0 to $j=n+2$, and canceling out all the repeated terms, we have

$$
\begin{aligned}
& \frac{1}{6}[\rho(-1)-\rho(n+2)] \\
& =\frac{1}{24} G \delta t[\psi(n+2) \psi(n+3)+\psi(n+1) \psi(n+2) \\
& \quad-\psi(-1) \psi(-2)-\psi(0) \psi(-1)] .
\end{aligned}
$$

Recall that all node points with indices $j<1$ are inside the bulk liquid region, and all node points with indices $j>n$ are inside the bulk vapor region. As the fluid density variation within the single-phase regions is almost negligible, we have $\rho(-1)=\rho_{L}, \psi(0)=\psi(-1)=\psi(-2)=\psi\left(\rho_{L}\right)$, and $\rho(n+$ $2)=\rho_{V}, \quad \psi(n+1)=\psi(n+2)=\psi(n+3)=\psi\left(\rho_{V}\right)$. Equation (A6) eventually leads to

$$
\frac{1}{6} \rho_{L}+\frac{1}{12} G \delta t \psi^{2}\left(\rho_{L}\right)=\frac{1}{6} \rho_{V}+\frac{1}{12} G \delta t \psi^{2}\left(\rho_{V}\right)
$$


or

$$
c_{s}^{2} \rho_{L}+\frac{1}{2} c_{s}^{2} G \delta t \psi^{2}\left(\rho_{L}\right)=c_{s}^{2} \rho_{V}+\frac{1}{2} c_{s}^{2} G \delta t \psi^{2}\left(\rho_{V}\right) .
$$

The two sides of the above equation are just the hydrodynamic pressures in the liquid and vapor phases. Equation (A8) simply indicates that PP LB models always ensure that the hydrodynamic pressures of the two single-phase bulk regions are equal in a flat interface case at the steady state.

The mechanical stability condition in Eq. (12) can be also derived from Eq. (A5). After certain mathematical manipulation, Eq. (A5) can be rewritten as

$$
\begin{aligned}
\frac{1}{3} \rho(j-1)+\frac{1}{6} G \delta t \psi^{2}(j-1) \\
\quad+\frac{1}{12} G \delta t \psi(j-1)[\psi(j)+\psi(j-2)-2 \psi(j-1)] \\
=\frac{1}{3} \rho(j)+\frac{1}{6} G \delta t \psi^{2}(j)+\frac{1}{12} G \delta t \psi(j)[\psi(j-1) \\
\quad+\psi(j+1)-2 \psi(j)] .
\end{aligned}
$$

Recalling that $c_{s}^{2}=1 / 3$, and $p(j)=c_{s}^{2} \rho(j)+\frac{1}{2} G c_{s}^{2} \delta t \psi^{2}(j)$, the above equation is equivalent to

$$
\begin{aligned}
& p(j)+\frac{1}{12} G \delta t \psi(j)[\psi(j+1)+\psi(j-1)-2 \psi(j)] \\
& \quad=p_{0}=\mathrm{const},
\end{aligned}
$$

where the boundary conditions in the bulk region require

$$
\begin{aligned}
p_{0} & =p\left(\rho_{L}\right)=c_{s}^{2} \rho_{L}+\frac{1}{2} G c_{s}^{2} \delta \psi^{2}\left(\rho_{L}\right) \\
& =p\left(\rho_{V}\right)=c_{s}^{2} \rho_{V}+\frac{1}{2} G c_{s}^{2} \delta \psi^{2}\left(\rho_{V}\right) .
\end{aligned}
$$

Equation (A10) can be further expressed as

$$
\frac{12}{G \delta t} \frac{p_{0}-p(j)}{\psi(j)}=\psi(j+1)+\psi(j-1)-2 \psi(j) .
$$

Summing this equation over the interface and substituting the boundary conditions, we finally reach

$$
\sum_{j=0}^{n+1} \frac{p_{0}-p(j)}{\psi(j)}=0
$$

which is the discrete mechanical stability condition. When $p_{0}$ satisfying Eq. (A13) deviates from the saturated pressure at the bubble or dew point predicted by the Maxwell equal area rule, i.e., $\tilde{p}_{0}$, the thermodynamic inconsistency occurs.

Taylor expanding the right-hand side of Eq. (A10) at $j$ and truncating all the high-order derivative terms, i.e.,

$$
\frac{d^{2} \psi}{d x^{2}}(j) \approx \psi(j+1)+\psi(j-1)-2 \psi(j),
$$

Eq. (A10) becomes

$$
p(j)+\frac{1}{12} G \delta t \psi \frac{d^{2} \psi}{d x^{2}}(j) \approx p_{0}=\text { const. }
$$

Following Shan's derivation in Ref. [10] from now on, we eventually arrive at

$$
\int_{\rho_{V}}^{\rho_{L}}\left(p_{0}-p\right) \frac{\psi^{\prime}}{\psi} d \rho=0
$$

The detailed steps from Eq. (A15) to Eq. (A16) are straightforward and can be found in the literature; thus, they are not repeated here. The integral mechanical stability condition, Eq. (A16), turns out to be an extremely good approximation to its discrete counterpart, Eq. (A13). In fact, one can validate this argument easily by examining Eq. (A16) as

$$
\begin{aligned}
\int_{\rho_{V}}^{\rho_{L}} & \left(p_{0}-p\right) \frac{\psi^{\prime}}{\psi} d \rho \\
= & \int_{\psi_{V}}^{\psi_{L}}\left(p_{0}-p\right) \frac{1}{\psi} d \psi \\
\approx & \frac{p_{0}}{2} \sum_{j}\left[\frac{1}{\psi(j+1)}+\frac{1}{\psi(j)}\right][\psi(j+1)-\psi(j)] \\
& -\frac{1}{2} \sum_{j}\left[\frac{p(j+1)}{\psi(j+1)}+\frac{p(j)}{\psi(j)}\right][\psi(j+1)-\psi(j)],
\end{aligned}
$$

with the LB simulation results from the flat interface case. As long as the simulation uses the original Shan-Chen force and Guo et al.'s forcing scheme, no matter if the thermodynamic consistency is achieved or not, its results at the steady state would always lead to trivial outcomes (on the machine roundoff error level) following Eq. (A17). This indicates that the approximation in Eq. (A14) is close to exact. Benefiting from this feature, $r_{p}$ that enforces precise thermodynamic consistency in actual PP LB simulations can be directly computed from the integral form of the mechanical stability condition, which avoids tuning.

On the contrary, with Kupershtokh et al.'s definition of Shan-Chen force [19], Eq. (A10) is modified as

$$
\begin{aligned}
p(j) & +\frac{\beta}{12} G \delta t \psi(j)[\psi(j+1)+\psi(j-1)-2 \psi(j)] \\
& +\frac{1-\beta}{24} G \delta t\left[\psi^{2}(j+1)+\psi^{2}(j-1)-2 \psi^{2}(j)\right] \\
= & p_{0}=\text { const. }
\end{aligned}
$$

With Li et al.'s forcing scheme [8], Eq. (A10) becomes

$$
\begin{gathered}
p(j)+\frac{1}{12} G \delta t \psi(j)[\psi(j+1)+\psi(j-1)-2 \psi(j)] \\
+\frac{1}{6} G^{2} \sigma \delta t^{2}[\psi(j+1)-\psi(j-1)]^{2}=p_{0}=\text { const. }
\end{gathered}
$$

In order to derive the integral forms of the mechanical stability condition, we make the following approximations:

$$
\begin{aligned}
& \frac{d^{2} \psi^{2}}{d x^{2}}(j) \approx \psi^{2}(j+1)+\psi^{2}(j-1)-2 \psi^{2}(j), \\
& 2 \frac{d \psi}{d x}(j) \approx \psi(j+1)-\psi(j-1) .
\end{aligned}
$$

However, these approximations have turned out to be not accurate. As a result, the tuning parameters of those methods computed from the corresponding modified integral mechanical stability condition, i.e., Eq. (32), would still fail to achieve precise thermodynamic consistency in LB simulations. 


\section{APPENDIX B: CUBIC EOSS AND BRIEF INTRODUCTION OF SAMPLE MATLAB CODES}

\section{Cubic EOSs}

The cubic EOSs used in the study are listed here.

\section{a. van der Waals (vdW) EOS.}

$$
p_{\mathrm{EOS}}^{\mathrm{vdW}}(\rho)=\frac{\rho R T}{1-b \rho}-a \rho^{2}
$$

where $a=\frac{27 R^{2} T_{c}^{2}}{64 P_{c}}, b=\frac{R T_{c}}{8 P_{c}}, R$ is the specific gas constant, and $P_{c}$ and $T_{c}$ are the critical pressure and temperature of the pure substance, respectively.

\section{b. Carnahan-Starling (CS) EOS [24].}

$$
p_{\mathrm{EOS}}^{\mathrm{CS}}(\rho)=\rho R T \frac{1+b \rho / 4+(b \rho / 4)^{2}-(b \rho / 4)^{3}}{(1-b \rho / 4)^{3}}-a \rho^{2},
$$

where $a=0.4963 \frac{R^{2} T_{c}^{2}}{P_{c}}$ and $b=0.18727 \frac{R T_{c}}{P_{c}}$.

\section{c. Soave-Redlich-Kwong (SRK) EOS [25].}

$$
\begin{aligned}
p_{\mathrm{EOS}}^{S R K}(\rho) & =\frac{\rho R T}{1-b \rho}-\frac{a \alpha^{S R K}(T) \rho^{2}}{1+b \rho}, \\
\alpha^{S R K}(T) & =\left[1+\left(0.480+1.574 \omega-0.176 \omega^{2}\right)\left(1-\sqrt{T / T_{c}}\right)\right]^{2},
\end{aligned}
$$

where $a=0.42748 \frac{R^{2} T_{c}^{2}}{P_{c}}, b=0.08664 \frac{R T_{c}}{P_{c}}, \alpha^{S R K}$ is Soave's correction to the original Redlich-Kwong EOS, which is function of Pitzer's acentric factor $\omega$ and the reduced temperature
$T / T_{c} . \omega=0.344$ is the acentric factor for water, and $\omega=$ 0.0104 is the acentric factor for methane.

\section{d. Peng-Robinson (PR) EOS [26].}

$$
\begin{aligned}
p_{\mathrm{EOS}}^{P R}(\rho)= & \frac{\rho R T}{1-b \rho}-\frac{a \alpha^{P R}(T) \rho^{2}}{1+2 b \rho-b^{2} \rho^{2}}, \\
\alpha^{P R}(T)= & {[1+(0.37464+1.54226 \omega} \\
& \left.\left.-0.26992 \omega^{2}\right)\left(1-\sqrt{T / T_{c}}\right)\right]^{2},
\end{aligned}
$$

where $a=0.45724 \frac{R^{2} T_{c}^{2}}{P_{c}}, b=0.0778 \frac{R T_{c}}{P_{c}}$, and $\alpha^{P R}$ is the temperature correction of the PR EOS, which is again a function of the acentric factor and the reduced temperature.

\section{Brief introduction of sample MATLAB codes}

The first sample code in the Supplemental Material [20] uses the Newton-Raphson method to compute $r_{\rho}$ in the customized EOS to achieve full thermodynamic consistency with the amplitude of the customized van der Waals loop $\theta$ defined as Eq. (24). Given a reduced temperature $T_{r}$, and its corresponding $\tilde{\rho}_{L}, \tilde{\rho}_{V}$, and $\tilde{p}_{0}$ (all predicted by the Maxwell equal area rule), the code takes about $0.01 \mathrm{~s}$ on a personal computer to find $r_{\rho}$.

The other sample code [20] searches $r_{\theta}$ in the customized EOS to reproduce the desired surface tension and the corresponding $r_{\rho}$ to satisfy the thermodynamic consistency. This code uses the bisection method to search for $r_{\theta}$ and the Newton-Raphson method to find the corresponding $r_{\rho}$. Given a reduced temperature $T_{r}$, and its corresponding $\tilde{\rho}_{L}, \tilde{\rho}_{V}$, and $\tilde{p}_{0}$, the code takes about $30 \mathrm{~s}$ to find the targeted $r_{\theta}$ and $r_{\rho}$. Both sample codes use the PR EOS in LB units with $a=2 / 49, b=2 / 21, R=1$, and $\omega=0.344$, as suggested by Yuan and Schaefer [6].
[1] X. Shan and H. Chen, Lattice Boltzmann model for simulating flows with multiple phases and components, Phys. Rev. E 47, 1815 (1993).

[2] X. Shan and H. Chen, Simulation of nonideal gases and liquidgas phase transitions by the lattice Boltzmann equation, Phys. Rev. E 49, 2941 (1994).

[3] L. Chen, Q. Kang, Y. Mu, Y.-L. He, and W.-Q. Tao, A critical review of the pseudopotential multiphase lattice Boltzmann model: Methods and applications, Int. J. Heat Mass Transf. 76, 210 (2014).

[4] H. Huang, M. Sukop, and X. Lu, Multiphase Lattice Boltzmann Methods: Theory and Application (Wiley, New York, 2015).

[5] Q. Li, K. H. Luo, Q. Kang, Y. He, Q. Chen, and Q. Liu, Lattice Boltzmann methods for multiphase flow and phasechange heat transfer, Prog. Energy Combust. Sci. 52, 62 (2016).

[6] P. Yuan and L. Schaefer, Equations of state in a lattice Boltzmann model, Phys. Fluids 18, 042101 (2006).

[7] C. E. Colosqui, G. Falcucci, S. Ubertini, and S. Succi, Mesoscopic simulation of non-ideal fluids with self-tuning of the equation of state, Soft Matter 8, 3798 (2012).
[8] Q. Li, K. H. Luo, and X. Li, Forcing scheme in pseudopotential lattice Boltzmann model for multiphase flows, Phys. Rev. E 86, 016709 (2012).

[9] X. He and G. D. Doolen, Thermodynamic foundations of kinetic theory and lattice Boltzmann models for multiphase flows, J. Stat. Phys. 107, 309 (2002).

[10] X. Shan, Pressure tensor calculation in a class of nonideal gas lattice Boltzmann models, Phys. Rev. E 77, 066702 (2008).

[11] Q. Li and K. H. Luo, Achieving tunable surface tension in the pseudopotential lattice Boltzmann modeling of multiphase flows, Phys. Rev. E 88, 053307 (2013).

[12] A. Xu, T. Zhao, L. An, and L. Shi, A three-dimensional pseudopotential-based lattice Boltzmann model for multiphase flows with large density ratio and variable surface tension, Int. J. Heat Fluid Flow 56, 261 (2015).

[13] S. Khajepor and B. Chen, Multipseudopotential interaction: A consistent study of cubic equations of state in lattice Boltzmann models, Phys. Rev. E 93, 013303 (2016).

[14] S. F. Kharmiani, H. Niazmand, and M. Passandideh-Fard, An alternative high-density ratio pseudo-potential lattice 
Boltzmann model with surface tension adjustment capability, J. Stat. Phys. 175, 47 (2019).

[15] S. Gong and P. Cheng, Numerical investigation of droplet motion and coalescence by an improved lattice Boltzmann model for phase transitions and multiphase flows, Comput. Fluids 53, 93 (2012).

[16] Z. Guo, C. Zheng, and B. Shi, Discrete lattice effects on the forcing term in the lattice Boltzmann method, Phys. Rev. E 65, 046308 (2002).

[17] C. Peng, L. F. Ayala, O. M. Ayala, and L.-P. Wang, Isotropy and spurious currents in pseudo-potential multiphase lattice Boltzmann models, Comput. Fluids 191, 104257 (2019).

[18] H. B. Callen, Thermodynamics and an Introduction to Thermostatistics, 2nd Edition (John Wiley \& Sons, Inc., New York, 1985).

[19] A. Kupershtokh, D. Medvedev, and D. Karpov, On equations of state in a lattice Boltzmann method, Comput. Math. Appl. 58, 965 (2009).

[20] See Supplemental Material at http://link.aps.org/supplemental/ 10.1103/PhysRevE.101.063309 for the sample MATLAB code 1 to find the optimal $\tilde{\rho}_{M}$ in the customized EOS with the given $\theta$ by Eq. (24) to achieve thermodynamic consistency, and for the sample MATLAB code 2 to search $\theta$ and the corresponding $\tilde{\rho}_{M}$ in the customized EOS to reproduce the desired surface tension and achieve thermodynamic consistency.

[21] A. Hu, L. Li, S. Chen, Q. Liao, and J. Zeng, On equations of state in pseudo-potential multiphase lattice Boltzmann model with large density ratio, Int. J. Heat Mass Transf. 67, 159 (2013).

[22] A. Montessori, G. Falcucci, M. La Rocca, S. Ansumali, and S. Succi, Three-dimensional lattice pseudo-potentials for multiphase flow simulations at high density ratios, J. Stat. Phys. 161, 1404 (2015).

[23] C. D. Stiles and Y. Xue, High density ratio lattice Boltzmann method simulations of multicomponent multiphase transport of $\mathrm{H}_{2} \mathrm{O}$ in air, Comput. Fluids 131, 81 (2016).

[24] N. F. Carnahan and K. E. Starling, Equation of state for nonattracting rigid spheres, J. Chem. Phys. 51, 635 (1969).

[25] G. Soave, Equilibrium constants from a modified RedlichKwong equation of state, Chem. Eng. Sci. 27, 1197 (1972).

[26] D.-Y. Peng and D. B. Robinson, A new two-constant equation of state, Ind. Eng. Chem. Fundam. 15, 59 (1976). 